\title{
Modelización de los antecedentes y consecuentes de la comunicación integrada de marketing
}

\section{Modeling driving-factors and effects of Integrated Marketing Communications (IMC)}

\begin{abstract}
Lucía Porcu
Licenciada en Relaciones Públicas por la Università degli Studi di Udine, Italia luciap@correo.ugr.es

Salvador del Barrio García

Profesor Titular de Comercialización e Investigación de Mercados dbarrio@ugr.es
\end{abstract}

Facultad de Ciencias Económicas y Empresariales Universidad de Granada Campus de Cartuja s/n 18071 Granada Telf. 958242347 


\title{
Modelización de los antecedentes y consecuentes de la comunicación integrada de marketing
}

\author{
Modeling driving-factors and effects of Integrated Marketing \\ Communications (IMC)
}

\section{Resumen}

La comunicación de marketing desempeña un papel muy importante en la construcción $\mathrm{y}$ el mantenimiento de las relaciones con los stakeholders y en rentabilizar estas relaciones en términos de valor de marca y resultados financieros. A partir de la década de los noventa, la Comunicación Integrada de Marketing (CIM) ha atraído una considerable atención en la literatura de Marketing. Este trabajo de investigación se propone un modelo que, a través de la identificación de antecedentes y consecuentes de la CIM, aclara el funcionamiento del proceso de integración y su relación con los resultados financieros y de marca.

Palabras clave: Comunicación Integrada de Marketing, modelización, antecedentes, resultados de marca, resultados financieros.

\begin{abstract}
Marketing communication plays an important role in building and maintaining stakeholder relationships, and in leveraging these relationships in terms of brand equity and financial performance. Since the mid 1990s, the Integrated Marketing Communications (IMC) has attracted considerable attention in the literature.

This present research proposes a conceptual model based on the identification of antecedents and consequences of implementing the IMC paradigm, that seeks to provide insight into a range of questions related to understanding how the IMC process works and whether the successful implementations of IMC might result in more favourable brand and financial outcomes.
\end{abstract}

Keywords: Integrated Marketing Communication, modelling, driving-factors, effects, brand performance, financial performance. 


\section{INTRODUCCIÓN}

La comunicación de marketing, como relación sistemática entre una organización y su mercado, permite comunicar ideas y estimular una percepción determinada de sus productos y servicios a los distintos grupos de interés.

La variedad de herramientas de promoción de la que dispone una organización, así como los distintos canales, pueden ocasionar una cierta dispersión del mensaje. Cada una de esas herramientas ha tenido su desarrollo autónomo, que, a nivel práctico, ha desencadenado actuaciones independientes y no siempre coordinadas. Ello no presupone una comunicación contradictoria, pero desde luego la falta de una visión conjunta de los distintos instrumentos de comunicación sí que es un importante impedimento para la consecución de las deseadas sinergias de comunicación.

Tradicionalmente, incluso hoy en día se sigue haciendo, se ha distinguido entre dos categorías de herramientas de comunicación: above the line y below the line. La primera se refiere a la publicidad en medios masivos o convencionales (televisión, radio, revistas, publicidad exterior, etc.), mientras que la segunda categoría recoge las herramientas de comunicación en medios no masivos o convencionales e incluye las fuerzas de ventas, el marketing directo, la promoción de ventas, o las relaciones públicas, entre otras. Además, en los últimos años la utilización de Internet ha supuesto una nueva categoría autónoma, al principio identificada como no convencional, pero con el paso del tiempo considerada como un medio convencional más (Bigné, 2003).

La relevancia alcanzada por la comunicación de marketing se argumenta desde la evolución del propio concepto de marketing transaccional hacia el más reciente del marketing relacional. Grönroos (1996) resume la evolución del marketing como el paso de una lógica enfocada en la transacción y el producto a una lógica orientada en la construcción de relaciones duraderas y el desarrollo de recursos y competencias de cara a crear valor para el cliente. Duncan y Moriarty (1998) observan que muchos roles del marketing, sobre todo en el ámbito de los servicios, consisten fundamentalmente en posiciones donde la comunicación representa el elemento central de las actividades de marketing. Desde este prisma, la comunicación no sólo se genera por las actividades de marketing, sino que está presente en el corazón de todas las funciones de la empresa (you cannot not comunicate). Tradicionalmente, la comunicación ha sido conceptualizada y gestionada desde una perspectiva funcional con un claro objetivo persuasivo. La persuasión representa la comunicación en una sola vía y se identifica con la visión transaccional del marketing: informar, persuadir y recordar. En cambio, la comunicación desde una perspectiva relacional es un concepto mucho más amplio que no se queda en la mera persuasión sino que busca, a través de la interactividad, mantener un diálogo continuo con los stakeholders con tres claros objetivos: informar, escuchar y responder, al objeto de conseguir aumentar el valor de marca de la organización.

Schultz y Schultz (1998) consideran que está surgiendo la necesidad de una nueva perspectiva sobre cómo los programas de marketing y comunicación de marketing son planificados, desarrollados e implementados. Según estos autores, la integración de una amplia gama de actividades y funciones que influyen e impactan sobre el flujo dinámico de información entre las organizaciones y sus grupos de interés (stakeholders) puede proporcionar la solución. Ya no es relevante pensar en publicidad, relaciones públicas y promoción de ventas en términos de disciplinas independientes o departamentos estanco, ni repartir las actividades en above the line o below the line. 
No es apropiado limitar la responsabilidad de la comunicación de marketing sólo dentro de los canales de comunicación tradicionales. Todos éstos son asuntos y conceptos antiguos, que como señalan Schultz y Schultz (1998), no se ajustan a las necesidades de la organización del siglo XXI, ni a los clientes o consumidores del siglo XXI porque fueron desarrollados para un mercado y organizaciones que ya no existen y para un sistema de medios que ya no es el dominante.

Los cambios que afectan actualmente al mercado, a los consumidores/clientes, a la tecnología, a los medios y a la habilidad de gestionar y almacenar datos, se complementan para crear una situación de transición en la que la mayoría de los profesionales de marketing se encuentran actualmente. Es la innovación tecnológica la que nos ha conducido hacia la necesidad de integración. Schultz (1996), en su trabajo titulado Inevitabilidad de la Integración, considera que el debate acerca de integración o no de la comunicación en las organizaciones no tiene mucho sentido ya que en realidad es inevitable que el consumidor integre todos los mensajes que recibe de una organización. Es decir que la integración sólo depende del consumidor y tiene lugar incluso cuando los profesionales de marketing y de publicidad no la prevén. Así que también en el caso en que la empresa no evite el envío de mensajes totalmente descoordinados los consumidores los agregarán e integrarán utilizando algún patrón. Pero desafortunadamente, en este caso, el criterio elegido por el consumidor para realizar tal integración puede causar un grave daño para el valor de la marca.

Así pues, dado que la integración ocurre incluso cuando la empresa no la planifica y es inevitable, ésta tiene que ser capaz de gestionarla según le convenga. Lo mejor que los profesionales pueden hacer en este contexto es intentar entender el proceso de integración y modificar sus enfoques y conceptos para maximizar la rentabilidad de la integración, y conseguir así los esperados beneficios resultantes de la obtención de sinergias de comunicación. La comunicación integrada de marketing (CIM), supone una alternativa coherente, orientada al consumidor, que puede remplazar los enfoques de marketing más tradicionales centrados en la transacción por un enfoque relacional basado en la comunicación (Stephens, Hill y Bergman, 1996). En una época de mercados saturados, con un continuo aumento de la competencia y dominado por consumidores sofisticados, los gestores de marketing tienen que trabajar más duro que nunca. Se requiere un enfoque centrado en la sinergia, en el que todas las actividades de marketing trabajen conjuntamente para enviar un mensaje coherente a los consumidores.

El enfoque CIM ha surgido recientemente para alcanzar los retos de un entorno cada vez más complejo al que el paradigma clásico de las 4 Ps no puede dar solución, y al que autores como Schultz, Tannenbaumm y Lauterborn (1994) proponen sustituirlo por otro denominado 4 Cs: consumidor, coste, conveniencia y comunicación, entendida ésta última como una interactividad continua con los stakeholders.

Este trabajo tiene como objetivo principal analizar desde una perspectiva holística el debate conceptual en torno a la CIM y a sus principales factores antecedentes y consecuentes. Todo ello nos permitirá plantear un modelo conceptual de funcionamiento de la CIM que ayude comprender en toda su dimensión lo que representa este nuevo paradigma del marketing dentro de la gestión empresarial contemporánea, las principales variables que la determinan o frenan, así como los principales beneficios que su implementación produce. 


\section{EVOLUCIÓN DEL CONCEPTO DE COMUNICACIÓN INTEGRADA DE MARKETING. UNA PROPUESTA INTEGRADORA}

Como muchas nuevas tendencias de la investigación en el ámbito del marketing y de la publicidad, el concepto de CIM fue lanzado y adoptado por los académicos en respuesta a los desarrollos profesionales de marketing y publicidad. Algunos autores han ido desarrollando en los últimos años el cuerpo teórico de la CIM con el objetivo de dirigir, describir y explicar las prácticas corrientes de comunicación de marketing que no han sido captadas por la comunicación de masas tradicional (Schultz et al., 1993; Schultz, 1996, 1999; Nowak y Phelps, 1994).

Al ser un ámbito de estudio relativamente reciente es lógico que aun en nuestros días el paradigma de la CIM carezca de claridad en cuanto a su definición y límites de aplicación. Los académicos y los profesionales han debatido durante más de una década acerca de este concepto sin llegar a un acuerdo definitivo sobre lo que es exactamente y los beneficios que ofrece a los profesionales de marketing. A lo largo de estos años, la CIM ha sido definida desde diferentes perspectivas, como una filosofía de gestión (Stewart, 1996), como un movimiento educativo (Hutton, 1996) o incluso como una práctica de negocio unificadora (Moriarty, 1993).

Nos proponemos en este epígrafe realizar un meta-análisis cualitativo de las definiciones más destacadas de la CIM hasta la fecha, al objeto de aportar claridad al debate conceptual del mismo, para lo cuál se clasificarán dichas definiciones en las tres categorías siguientes:

1) Enfoque 'inside-out'.

2) Enfoque 'outside-in'.

3) Enfoque estratégico interfuncional.

\subsection{El enfoque inside-out}

La primera categoría de conceptualización de la CIM prevé una pequeña, aunque muy leve, orientación al cliente, en la que la CIM representa una tarea relativamente sencilla consistente en juntar e integrar los elementos de la comunicación de marketing para que "hablen con una sola voz". Kitchen, et al. (2004) ponen como ejemplo de empresa que se encuentra en esta etapa a Cadbury, empresa británica líder en chocolate, que a través de una campaña promocional ofreció a sus clientes la posibilidad de obtener gratuitamente artículos deportivos a través del envío de envoltorios especiales de productos de su marca. Aunque la campaña estaba integrada en términos de publicidad, promoción de ventas, diseño del envoltorio y relaciones públicas, por otro lado, existía un desajuste en la mente de clientes, consumidores y expertos acerca de las conexiones entre chocolate y obesidad, y entre chocolate y las capacidades deportivas. Esta campaña, a pesar de pretender un beneficio para el consumidor, se clasificaría dentro de un enfoque 'inside-out'.

Representativa de este enfoque se encuentra una de las primeras definiciones sobre CIM, propuesta por la Northwestern University, que está entre las más citadas en la literatura y que fue acogida como propia por la American Association of Advertising Agencies en 1989: "un concepto de planificación de la comunicación de marketing que reconoce el valor añadido de un plan exhaustivo que evalúe el papel estratégico de una variedad de disciplinas de comunicación, como la publicidad tradicional, la promoción de ventas y las relaciones públicas...y combina estas disciplinas para facilitar claridad, coherencia y el máximo impacto de la comunicación" (Caywood et al., 1991; Schultz, 
1993). La debilidad de esta definición reside en el enfoque que considera a la CIM como una simple herramienta dirigida a juntar los elementos de comunicación para que 'hablen con una sola voz", pero que carece de una visión estratégica del conjunto de la empresa.

En esta misma línea, Duncan y Everett (1993) propusieron que la CIM consiste en la "coordinación estratégica de todos los mensajes y medios utilizados por una organización para influir en el valor de marca percibido", que si bien recoge el adjetivo 'estratégica' en realidad no supone un planteamiento bien distinto respecto a la definición anterior. Lo que la CIM promete, y necesita, es la emergencia de un nuevo paradigma que facilite llegar a una comunicación de marketing centrada claramente en los intereses del consumidor y del cliente. En esta definición, la CIM no parece proporcionar más que una promesa de todo esto. Si la CIM representa más que una simple coordinación de elementos de promoción, entonces la comunicación tiene que pasar de representar un componente táctico a ser un verdadero aliado estratégico de la empresa.

También Kotler (2000) propuso una definición que sigue la perspectiva 'insideout' definiendo a la CIM: "un concepto según el cual una empresa integra y cuidadosamente coordina sus variados canales de comunicación para conseguir un mensaje claro, coherente y convincente sobre la empresa y sus productos". En esta misma línea Percy, Rossiter y Elliot (2001), definen la CIM como la "planificación y ejecución de todos los tipos de comunicación de marketing necesarios para la marca, el servicio o la organización de cara a alcanzar unos objetivos de comunicación”.

En ámbito español, Bigné (2003) define a la CIM como "promoción integrada" haciendo hincapié en ella como herramienta táctica que facilita la coordinación entre las variables de promoción, si bien no considera adecuado reemplazar el clásico esquema del marketing mix por el de CIM. Más recientemente, Rodríguez (2006) presentó la CIM como 'una aproximación que conlleva un nuevo modo de concebir y llevar a cabo las comunicaciones con el mercado, que requiere una coordinación efectiva de los diferentes instrumentos de comunicación de marketing (venta personal, publicidad...) entre sí, con el resto de actividades de la empresa que también influyen en la imagen que se forman los consumidores sobre el producto o la marca de la organización". En su publicación, el autor pone énfasis en la distinción de los elementos del mix de comunicación, propia del enfoque tradicional, aunque sí reconoce la importancia de coordinar las distintas herramientas promocionales de cara a maximizar los efectos de la comunicación de marketing.

En parte, el obstáculo en la definición de la CIM ha venido como consecuencia de la rápida evolución del concepto y su dispar aplicación por parte de las empresas. Al principio, éstas se centraron en las operaciones y tácticas de la comunicación de marketing, mientras que posteriormente comenzaron a darse cuenta de la necesidad de un enfoque más exhaustivo y holístico para construir relaciones con el cliente de cara a justificar sus acciones a la dirección.

\subsection{El enfoque outside-in}

La segunda categoría incluye las definiciones que representan el primer intento de pensar activamente en lo que clientes y consumidores quieren oír o ver, cuándo y dónde y a través de qué medios. Esto representa ya un enfoque de 'outside-in marketing'. Constituye una importante mejora con respecto a la primera etapa y 
potencialmente evita muchos de los errores que los expertos de marketing cometían con el enfoque 'inside-out'. Según Kitchen y Schultz (1999), sólo el 25\% de las empresas basa sus actividades de comunicación de marketing en una profunda comprensión de las dinámicas de su segmento de mercado. Schultz (1993) reconoce la necesidad de superar la fase 'inside-out', afirmando que "la CIM es el proceso de desarrollo e implementación de distintas formas de programas de comunicación persuasiva con clientes. Su objetivo es condicionar de forma directa el comportamiento del público seleccionado para las comunicaciones... es un proceso que empieza con el consumidor y funciona para determinar y definir cómo se deben desarrollar los programas de comunicación persuasiva".

En este sentido, Schultz y Schultz (1998) proponen una definición con la que pretenden captar el actual y futuro reto al que se enfrenta la CIM: "proceso estratégico de negocio usado para planificar, desarrollar, ejecutar y evaluar programas de comunicación de marca coordinados, cuantificables, persuasivos a lo largo del tiempo con consumidores, clientes, clientes potenciales y otros públicos internos y externos relevantes". La característica más importante de esta definición es que por primera vez aparece el enfoque en un 'proceso de negocio', un aspecto que lleva a crear sistemas en cadena a través del cual todas las actividades pueden ser planificadas, implementadas y evaluadas. Es un sistema dirigido por los datos, facilita una comprensión de las percepciones del cliente y de los contactos de marca y, lo más importante, provee una metodología subyacente sobre la cual todas las decisiones de inversión en comunicación pueden ser evaluadas, puesto que se centra en una evaluación del valor potencial y actual de consumidores y clientes para la organización.

Por su parte, Pickton y Broderick (2001) propusieron una de las definiciones de CIM más recientes y mejor planteadas a nivel académico y didáctico: "proceso que implica la gestión y la organización de todos los 'agentes' en el análisis, la planificación, la implementación y el control de todos los contactos - es decir, según los autores, 'cualquier comunicación personal o impersonal entre los miembros del público objetivo seleccionado y la empresa- medios, mensajes y herramientas promocionales de comunicación de marketing enfocados en públicos-objetivo seleccionados de manera que deriven en una mejor economía, eficiencia, eficacia y coherencia para lograr los objetivos de comunicación de marketing corporativo y de productos determinados". De la misma se desprenden las principales notas características de la estrategia de comunicación integrada:

- Identifica los objetivos de la comunicación de marketing con otros objetivos organizacionales.

- Es un proceso planificado que involucra todas las herramientas de comunicación de marketing de una forma coherente y sinérgica.

- Alcanza no sólo a los consumidores, sino incluye a todos los públicosobjetivo seleccionados por la organización: empleados, accionistas, proveedores, clientes, distribuidores, proscriptores, etc.

- Dirige todas las formas de contacto que formen parte de la comunicación de marketing. Esto implica cualquier comunicación relevante que surja de los contactos acaecidos dentro de la organización y/o entre la organización y sus públicos.

- Dirige e integra de manera efectiva todas las actividades promocionales y a todas las personas implicadas en las mismas. 
- Incluye todos los esfuerzos de comunicación de marketing corporativos y de producto/marca.

- Alcanza a todas las herramientas de comunicación ya sean personales o impersonales.

- Todos los mensajes corporativos o de productos/marcas deben basarse en una estrategia consistente y común que evite las inconsistencias entre ellos.

- Cualquier medio de comunicación es igual de adecuado para transmitir los mensajes comunicativos de la empresa.

Fill (2002) reafirmó la idea de una comunicación coherente y de un desarrollo estratégico al considerar la CIM como "un proceso de gestión que refuerza las propuestas de marca”. En este caso la CIM ya no es considerada sólo un proceso relacionado con la comunicación, sino también con la gestión y la marca.

De manera similar, Lagioni (2004) propone la siguiente definición de CIM: "optimización de los mensajes que se dirigen a los interlocutores de negocios uno por uno, que hay que percibir como valores complementarios de sus expectativas de base". En esta definición aparece el término 'optimización' (y no coordinación), que confiere el rigor, casi matemático, a los mensajes emitidos, más que a los medios utilizados, a interlocutores de negocios. En la literatura los autores a menudo han definido la CIM como la 'coordinación' de medios y mensajes, sin embargo el carácter que más diferencia la definición de Lagioni (2004) de las anteriores es su enfoque en los interlocutores one-by-one, es decir tomados uno por uno. Peppers y Rogers (2001) han escrito acerca del cliente one-to-one y sucesivamente adoptaron un enfoque en el cliente one-at-a-time. La difusión de las marcas y la búsqueda de cuotas de mercado cada vez más grandes conllevaron el alejamiento de la aplicación del enfoque de la comunicación de masas. Con la locución "one-to-one”, Peppers y Rogers (2001) quisieron hacer hincapié en la evolución hacia formas de marketing cada vez más personalizadas. En cambio, Lagioni (2004) con su enfoque “one-by-one” entiende que la comunicación toma en consideración a los interlocutores uno a la vez, incluyendo la posibilidad de identificarlos a través de los mecanismos que la informática pone a disposición. La locución "one-by-one" parte del supuesto según el cual la empresa instaura con sus interlocutores una relación interactiva, y este aspecto la diferencia de la locución "oneto-one". Del enfoque "one-by-one" deriva otra importante característica de la definición de Lagioni (2004): los mensajes a doble vía. La CIM existe sólo si produce una respuesta al mensaje enviado, una reacción de los distintos interlocutores (informar, escuchar y responder).

\subsection{El enfoque estratégico interfuncional}

Este enfoque de conceptualización va más allá de la yuxtaposición de los elementos de la promoción o del uso de la investigación de mercados. Se basa en la inversión de recursos significativos en la construcción de bases de datos segmentadas y en la reestructuración de la organización para que ésta consiga llegar a estar verdaderamente orientada a los clientes. En este contexto, la CIM supone un cambio real y una importante inversión de la organización. Sólo si los recursos de comunicación son invertidos y medidos con respecto al comportamiento real del cliente será posible estimar los beneficios en términos financieros. Duncan (2002) desarrolló un modelo sobre el proceso de CIM en el que ésta difiere de otros procesos centrados en el consumidor y cuyo fundamento es la comunicación. Este autor ofrece una nueva 
definición de CIM relativa al modelo propuesto: "proceso para gestionar las relaciones con el cliente que conducen al valor de marca. Más especificamente, [la CIM] es un proceso interfuncional con el objetivo de crear y alimentar relaciones rentables con los clientes y otros grupos de interés a través del control estratégico de todos los mensajes enviados a estos públicos e impulsando un diálogo intencional con ellos, basado en bases de datos".

Aquí no se trata ya de un proceso lineal, sino uno circular que representa el centro de todas las relaciones y crea valor de marca en forma de ventas, rentabilidad de cara a captar, retener y aumentar el número de clientes. El concepto de 'proceso interfuncional' implica que todos los departamentos y las agencias externas tienen que trabajar al unísono en la planificación y gestión de las fases de las relaciones de marca. Creando y alimentando las relaciones con los stakeholders, se captan nuevos clientes con los que se establece una interacción que permitirá encontrar las maneras de satisfacer sus necesidades y deseos. Es importante destacar la idea de 'rentabilidad' de las relaciones con el cliente, puesto que no todas las relaciones tienen el mismo valor para la empresa. El control estratégico de todos los mensajes se refiere a todos los aspectos del marketing mix.

Duncan y Mulhern (2004) sugieren que la CIM debe ser más estratégica que ejecutiva, ir más allá de los mensajes de promoción de ventas y de publicidad, incluir la comunicación de doble vía y ser dirigida por los resultados. Todo esto les lleva a una redefinición de la CIM como "proceso interfuncional continuo e interactivo de planificación, ejecución y control de marca que integra todas las partes en el proceso de intercambio en orden a maximizar la satisfacción de las necesidades y deseos de cada uno de los grupos". Esta redefinición refleja el cambio de perspectiva, pasando a considerar la gestión de la comunicación de marketing más como una serie de procesos que cruzan los límites tradicionales de los departamentos, empleando el conocimiento y las destrezas de especialistas y no especialistas en comunicación (Cornelissen, 2001).

A pesar de la considerable atención de la que puede presumir la CIM en la literatura en los últimos diez años, todavía sigue vivo el debate que hace de la CIM un rígido conjunto de especificaciones y reglas. Sin embargo, algunos autores sugieren que hay que considerar la CIM como una filosofía, cuya implementación dependerá de cada situación específica, para que así tenga la flexibilidad necesaria para adaptarse a los cambios del mercado (Gould, 2004; Phelps y Johnson, 1996; Lee y Park, 2007).

Gould (2004), por su parte, apoya la visión de la CIM como un concepto teórico controvertido en términos de generalización de lo que es y lo que realmente hace. Sin embargo, si se considera la CIM desde otra perspectiva paradigmática en la teoría, llamada 'post-estructuralismo', entonces se puede ver la CIM como un "conjunto de prácticas y discursos, que dependen del contexto, donde las interpretaciones de determinados profesionales localizados son tan importantes como las interpretaciones teoréticas".

Más recientemente Christensen, Firat y Torp (2008) hablan únicamente de comunicación integrada (CI) y la definen como "la noción y la práctica de alineación de los símbolos, procesos y comportamientos de cara a que una empresa comunique con claridad, coherencia y continuidad en y a lo largo de los límites formales de la empresa". Sin la alineación de los símbolos y mensajes las empresas reducen su impacto potencial e infra-optimizan sus presupuestos de comunicación (Smith, 1996). Sin claridad y consistencia en sus comunicaciones, las empresas tienen dificultades en seguir adelante como marcas distintivas e interesantes en un mercado competitivo 
(Duncan, 2005; Shimp, 2003). Sin coherencia entre los mensajes, procesos y comportamientos, las empresas no podrían pretender ser identificadas como legítimas protagonistas en el mundo globalizado actual (Schultz y Kitchen, 2000).

El concepto CI tal y como lo plantean Christensen et al. (2008) es más amplio que el de CIM, puesto que este último tiende a asumir que la integración tiene lugar, o debe hacerlo, en el ámbito del marketing. Hoy en día, la CI es una disciplina más amplia que conecta el marketing a otras disciplinas, incluyendo el diseño corporativo, la cultura de empresa, las relaciones públicas y la comunicación corporativa (Christensen et al., 2008). Conjuntamente, las disciplinas de marketing, las relaciones públicas y la comunicación corporativa están promoviendo una visión de una empresa que es capaz de analizar y controlar su propia comunicación como entidad coherente. Mientras los primeros conceptos de CIM se centraban en la coordinación de un conjunto limitado de parámetros de comunicación para obtener mensajes claros y coherentes, el tipo de integración propuesto por Christensen et al. (2008) involucra a la empresa a través de la inclusión de empleados, clientes y otros stakeholders. Considerando que las empresas contemporáneas comunican con todo lo que hacen o dicen, la comunicación integrada se ha desarrollado a partir de una actividad especializada hasta llegar a ser una cuestión que interesa toda la empresa (Duncan y Moriarty, 1998; Gronstedt, 1996).

Tabla 1. Evolución teórica del paradigma de la CIM

\begin{tabular}{|c|c|}
\hline $\begin{array}{l}\text { AMERICAN ASSOCIATION } \\
\text { OF ADVERTISING } \\
\text { AGENCIES (1989) }\end{array}$ & $\begin{array}{l}\text { Un concepto de la planificación de comunicación de Marketing que reconoce el valor } \\
\text { añadido de un plan exhaustivo que estima los roles estratégicos de una variedad de } \\
\text { disciplinas y combina estas disciplinas para proveer claridad, coherencia y el máximo } \\
\text { impacto de la comunicación. }\end{array}$ \\
\hline $\begin{array}{l}\text { CAYWOOD, SCHULTZ Y WANG } \\
\text { (1991) }\end{array}$ & $\begin{array}{l}\text { Un concepto de planificación de la comunicación de marketing que reconoce el valor } \\
\text { añadido de un plan exhaustivo que evalúe el papel estratégico de una variedad de } \\
\text { disciplinas de comunicación, como la publicidad tradicional, la promoción de ventas y } \\
\text { las relaciones públicas... y combina estas disciplinas para facilitar claridad, coherencia } \\
\text { y el máximo impacto de la comunicación. }\end{array}$ \\
\hline SCHULTZ, (1993) & $\begin{array}{l}\text { El proceso de desarrollo e implementación de distintas formas de programas de } \\
\text { comunicación persuasiva con clientes y stakeholders. Su objetivo es influir o } \\
\text { directamente condicionar el comportamiento del target seleccionado para las } \\
\text { comunicaciones. }\end{array}$ \\
\hline DUNCAN Y EVERETT (1993) & $\begin{array}{l}\text { La coordinación estratégica de todos los mensajes y medios utilizados por una } \\
\text { organización para influir en el valor de marca percibido. }\end{array}$ \\
\hline KOTLER (2000) & $\begin{array}{l}\text { Un concepto según el cual una empresa integra y cuidadosamente coordina sus } \\
\text { variados canales de comunicación para conseguir un mensaje claro, coherente y } \\
\text { convincente sobre la empresa y sus productos. }\end{array}$ \\
\hline $\begin{array}{l}\text { PERCY, ROSSITER Y ELLIOT } \\
(2001)\end{array}$ & $\begin{array}{l}\text { Planificación y ejecución de todos los tipos de comunicación de marketing necesarios } \\
\text { para la marca, el servicio o la organización de cara a alcanzar unos objetivos de } \\
\text { comunicación. }\end{array}$ \\
\hline BIGNÉ (2003) & $\begin{array}{l}\text { Un enfoque que permite la identificación del mensaje o mensajes... y su transmisión a } \\
\text { través de las diversas herramientas de forma coordinada de modo que se maximicen } \\
\text { los esfuerzos y se obtenga un impacto mayor y nítido entre los diversos públicos a los } \\
\text { que se dirige la marca o empresa. }\end{array}$ \\
\hline RODRÍGUEZ (2006) & $\begin{array}{l}\text { Una aproximación que conlleva un nuevo modo de concebir y llevar a cabo las } \\
\text { comunicaciones con el mercado, que requiere una coordinación efectiva de los }\end{array}$ \\
\hline
\end{tabular}




\begin{tabular}{|c|c|}
\hline & $\begin{array}{l}\text { diferentes instrumentos de comunicación de marketing (venta personal, publicidad...) } \\
\text { entre sí, con el resto de actividades de la empresa que también influyen en la imagen } \\
\text { que se forman los consumidores sobre el producto o la marca de la organización. }\end{array}$ \\
\hline \multicolumn{2}{|l|}{ Enfoque Outside-in } \\
\hline SCHULTZ (1993) & $\begin{array}{l}\text { El proceso de desarrollo e implementación de distintas formas de programas de } \\
\text { comunicación persuasiva con clientes. Su objetivo es influir o directamente } \\
\text { condicionar el comportamiento del target seleccionado para las comunicaciones. La } \\
\text { CIM considera que, en todos los contactos de la empresa o marca con un cliente, el } \\
\text { producto o el servicio son potenciales canales de distribución para futuros mensajes. }\end{array}$ \\
\hline SCHULTZ Y SCHULTZ, (1998) & $\begin{array}{l}\text { Un proceso estratégico de negocio usado para planificar, desarrollar, ejecutar y } \\
\text { evaluar programas de comunicación de marca coordinados, cuantificables, } \\
\text { persuasivos a lo largo del tiempo con consumidores, clientes, clientes potenciales y } \\
\text { otros públicos internos y externos relevantes. }\end{array}$ \\
\hline PICKTON Y BRODERICK, (2001) & $\begin{array}{l}\text { Un proceso que implica la gestión y la organización de todos los 'agentes' en el } \\
\text { análisis, la planificación, la implementación y el control de todos los contactos } \\
\text { (cualquier comunicación personal o impersonal entre los miembros del público } \\
\text { objetivo seleccionado y la empresa) medios, mensajes y herramientas promocionales } \\
\text { de comunicación de marketing enfocados en targets seleccionados de manera que } \\
\text { deriven en una mejor economía, eficiencia, eficacia y coherencia para lograr los } \\
\text { objetivos de comunicación de marketing corporativo y de productos determinados. }\end{array}$ \\
\hline LAGIONI (2004) & $\begin{array}{l}\text { La optimización de los mensajes que se dirigen a los interlocutores de negocios, } \\
\text { tomados uno por uno, que hay que percibir como valores complementarios de sus } \\
\text { expectativas de base. }\end{array}$ \\
\hline FILL (2002) & Un proceso de gestión que refuerza las propuestas de marca. \\
\hline \multicolumn{2}{|c|}{ Enfoque estratégico interfuncional } \\
\hline DUNCAN Y MORIARTY (1998) & $\begin{array}{l}\text { Un proceso sistémico que requiere determinados elementos de soporte a nivel } \\
\text { corporativo, de comunicación de marketing y de marketing. }\end{array}$ \\
\hline DUNCAN (2002) & $\begin{array}{l}\text { Un proceso para gestionar las relaciones con el cliente que conducen al valor de } \\
\text { marca... Un proceso interfuncional con el objetivo de crear y alimentar relaciones } \\
\text { rentables con los clientes y otros grupos de interés a través del control estratégico de } \\
\text { todos los mensajes enviados a estos públicos e impulsando un diálogo intencional con } \\
\text { ellos, basado en bases de datos. }\end{array}$ \\
\hline DUNCAN Y MULHERN (2004) & $\begin{array}{l}\text { Un proceso interfuncional continuo e interactivo de planificación, ejecución y control } \\
\text { de marca que integra todas las partes en el proceso de intercambio en orden a } \\
\text { maximizar la satisfacción de las necesidades y deseos de cada uno de los grupos. }\end{array}$ \\
\hline GouLD (2004) & $\begin{array}{l}\text { Un conjunto de prácticas y discursos, que dependen del contexto, donde las } \\
\text { interpretaciones de determinados profesionales localizados son tan importantes como } \\
\text { las interpretaciones teoréticas. }\end{array}$ \\
\hline $\begin{array}{l}\text { CHRISTENSEN, FIRAT Y TORP } \\
(2008)\end{array}$ & $\begin{array}{l}\text { Noción y práctica de alineación de los símbolos, procesos y comportamientos de cara } \\
\text { a que una empresa comunique con claridad, coherencia y continuidad en y a lo largo } \\
\text { de los límites formales de la empresa. }\end{array}$ \\
\hline
\end{tabular}

Fuente: Elaboración Propia

\subsection{Propuesta de definición de la comunicación integrada de marketing.}

La falta de una definición universalmente aceptada es la barrera más fuerte para desarrollar herramientas significativas de medición de la CIM. Ésta no se puede medir 
sin antes especificar qué representa en realidad dentro de la gestión de las empresas y qué dimensiones la componen. Sus mayores defensores (Rapp y Collins, 1988, 1990; Roman, 1988; Stevenson, 1989; Schultz et al., 1993, Duncan y Moriarty, 1998) la perciben como un fenómeno que lleva a una reestructuración global de los papeles del marketing y de la comunicación, a través de la eliminación de los muros que existen entre las principales áreas de comunicación de marketing (publicidad, promoción de ventas, relaciones públicas, etc.).

Como evidencia de este fenómeno, el profesional de marketing aumenta su demanda de campañas multidisciplinares, con estrategias de publicidad que cumplan objetivos de comunicación y de comportamiento simultáneamente, y ejecuciones creativas que generen a la vez notoriedad, promuevan las posiciones de marketing, impulsen respuestas de comportamiento inmediatas y construyan bases de datos de clientes que puedan ser utilizadas de cara a alimentar relaciones a largo plazo con el cliente.

La importancia de una definición formal de la CIM se ha puesto de manifiesto en muchas publicaciones académicas (Duncan, 2002; Fill, 2002; Kitchen y Schultz, 1999; Schultz, Tannenbaumm y Lauterborn, 1994), sin embargo, hasta la fecha se ha hecho aun poco por solucionar la incertidumbre que rodea el concepto teórico de CIM (Kitchen y Schultz, 1997, 1999, 2000).

Tras la revisión de la literatura se propone una definición de CIM que aclare a los investigadores dónde se encuentra este paradigma actualmente y qué papel ha de desempeñar en la gestión actual empresarial, en sentido amplio, y no sólo como herramienta para la gestión de la comunicación de marketing. Así, definimos la CIM como aquel proceso interactivo y sistémico de planificación interfuncional y optimización de los mensajes enviados a todos los grupos de interés de cara a comunicar con coherencia $\mathbf{y}$ transparencia al objeto de conseguir sinergias y fomentar relaciones rentables a largo plazo. Esta definición recoge el carácter multidimensional del concepto, distinguiéndose cuatro dimensiones principales:

1) One voice (una sola voz). Se trata de la esencia de la integración, puesto que implica lograr y mantener una imagen, una posición, y un mensaje claro y coherente a través de todas las herramientas de la comunicación de marketing (Nowak y Phelps, 1994; Phelps y jonson, 1996). Por este motivo, una estrategia común tiene que unificar la actividad de las relaciones públicas, la publicidad, la promoción de ventas, el marketing directo e interactivo, etc.

2) Interactividad. La CIM es una comunicación de doble vía (two-ways symmetric), porque a través de este paradigma se pretende mantener un diálogo continuo entre la empresa y sus interlocutores, que no incluye exclusivamente a los clientes. La innovación tecnológica desempeña aquí un papel muy relevante, sin el cual carecería de sentido este nuevo paradigma. El marketing relacional no ha sido capaz de integrar, ni en la teoría ni en la práctica, el verdadero valor que juega la comunicación en la construcción de relaciones con los clientes y con los stakeholders. Se ha centrado en variables como el compromiso y la confianza que son en realidad producto de la 'comunicación'. Como afirman Duncan y Moriarty (1998) la interactividad es la pieza que le faltaba a la teoría del marketing relacional para conseguir construir auténticas y verdaderas relaciones con los públicos de interés a través de un contacto continuo con su entorno escuchándole y respondiéndole. 
3) Planificación interfuncional. En la comunicación de marketing tradicional, el punto de referencia es el marketing mix, en cambio bajo este nuevo paradigma es la empresa en su totalidad. La integración es una ventaja competitiva que es capaz de optimizar la relación entre mensajes, medios y receptores y no simplemente la coordinación de los elementos del marketing mix. Las implicaciones son muy importantes dado que todo lo que hace, o no hace, la empresa comunica y no sólo el departamento de marketing. La CIM debe por tanto alentar una planificación interfuncional y promover la empresa in toto. Además, todos los colaboradores de la misma tendrán que adquirir una orientación a la comunicación.

4) Relaciones rentables a largo plazo. Duncan y Moriarty (1998) afirmaron que la comunicación interactiva a nivel corporativo, de marketing y de comunicación de marketing, lleva a relaciones a largo plazo que conllevan un aumento del valor de marca. Puesto que la CIM debe ser una herramienta fundamentalmente estratégica, ésta debe proponerse la consecución de objetivos a largo plazo.

En la definición propuesta se apuesta por la "optimización" frente a la "coordinación", dado que este término confiere un valor más riguroso al tipo de mensajes transmitidos, más que a los medios utilizados, a los interlocutores de la organización (stakeholders). Los medios representan únicamente los vehículos de los mensajes, mientras que los mensajes contienen los valores que la empresa quiere transmitir a sus interlocutores. En esta definición se hace hincapié en el valor de los mensajes, puesto que el problema está en la selección de los mismos y su adaptación al público objetivo.

Un elemento común de las definiciones más recientes parece ser la representación de la CIM como proceso, en una dúplice dimensión 'táctica' y 'estratégica'. Con 'dimensión estratégica' de la gestión de marketing se entiende el marco que provee la guía para emprender las acciones (o tácticas) que deben ponerse en marcha y, al mismo tiempo, formada por las acciones implementadas y las respuestas que competidores, clientes y otros grupos de interés producen. En un sentido más amplio, el enfoque estratégico pone énfasis en la identificación adecuada de las oportunidades del mercado como base para la planificación de marketing y el crecimiento, con el objetivo de conseguir una ventaja competitiva sostenible. La dimensión táctica, en cambio, hace referencia a las actividades a corto plazo que hay que poner en marcha en la implementación de determinadas estrategias para conseguir los objetivos de la planificación de marketing.

En definitiva, con la definición propuesta, se pretende hacer hincapié en los componentes estratégicos de la CIM, como son la planificación basada en el mercado y en las expectativas financieras (Duncan y Moriarty, 1997, 1998; Duncan y Mulhern, 2004; Kitchen, Brignell, Li y Spickett-Jones, 2004; Low, 2000, Schultz,1998), la integración interfuncional (Duncan y Mulhern, 2004; Cornelissen, 2001; Duncan y Moriarty, 1997, 1998; Phelps y Johnson, 1996; Smith, 1996) el compromiso para la inversión de recursos en la CIM (Duncan y Moriarty, 1997, 1998; Eagle y Kitchen, 2000; Smith, 1996) la interactividad y conectividad con clientes y grupos de interés (Duncan y Moriarty , 1997, 1998; Pickton y Hartley , 1998; Schultz, 1998; Smith, 1996) y la coherencia estratégica (Duncan y Moriarty, 1997, 1998; Cornelissen, 2001; Eagle y Kitchen, 2000; Low, 2000; Pickton y Hartley, 1998; Schultz, 1998; Smith, 1996; Phelps y Johnson, 1996). 


\section{MODELIZACIÓN DE LOS ANTECEDENTES Y CONSECUENTES DE LA COMUNICACIÓN INTEGRADA DE MARKETING}

Una vez delimitado el concepto de CIM y analizadas sus dimensiones componentes, a continuación, se examinan los principales antecedentes y consecuentes del mismo al objeto de plantear un modelo teórico de funcionamiento de la CIM.

\subsection{Antecedentes de la CIM}

La revisión de los factores que impulsan u obstaculizan el logro de un determinado nivel de integración permite agruparlos en dos grandes grupos: antecedentes endógenos y exógenos.

\subsubsection{Antecedentes endógenos}

El primer grupo incluye los factores relacionados con la alta dirección, la dinámica interdepartamental, los sistemas organizativos, el tamaño de la empresa y su sector de actividad y otras variables relacionadas con la gestión empresarial.

\section{FACTORES RELACIONADOS CON LA ALTA DIRECCIÓN}

Dentro de éstos cabe distinguir a su vez los siguientes:

a. Compromiso con la aplicación de la CIM. Éste ha sido identificado por distintos autores como uno de los aspectos que más influyen en la gestión del proceso de la CIM. Su compromiso implica que los directivos garanticen coherencia entre lo que dicen y lo que hacen, de cara a evitar ambigüedad para los directivos intermedios que puede resultar muy dañina para la deseada integración. Smith (1996) considera que una ausencia general de compromiso con la comunicación integrada a nivel de alta dirección a menudo conlleva un aislamiento estructural de las herramientas comunicativas y de los directivos de comunicación. La implicación de la alta dirección en la planificación de la CIM significa conceder toda la facilidad y flexibilidad posible para conseguir la integración interfuncional y resolver los conflictos internos e interdepartamentales (Duncan y Everett, 1993; Eagle y Kitchen, 1999).

b. Algunos autores sugieren que la actitud de los directivos al cambio tiene una influencia positiva en la CIM. El equipo directivo debe tener la voluntad de cambiar las políticas de empresa que inhiben la implementación de la CIM (Phelps, Harris y Jonhson, 1996; Smith, 1996; Duncan y Mulhern, 2004).

c. Asimismo, es igual de importante para favorecer la CIM la tolerancia al riesgo del equipo directivo, puesto que la adopción des este concepto exige la disposición de la dirección a asumir el riesgo inherente al seguimiento de mercados cambiantes (Phelps et al., 1996).

Muchos estudiosos de la CIM consideran este antecedente como un aspecto estructural, es decir, de responsabilidad formal y de líneas de mando (Schultz y Schultz, 2003). En particular, Schultz (1993) considera que es necesario elevar la responsabilidad de gestionar los mensajes y símbolos hasta la punta de la pirámide organizativa, es decir hasta el top management. Schultz y Kitchen (2000) enfatizan que la necesidad de control del proceso entero de comunicación de marketing desde una localización central es incluso más importante en un contexto global. Este sistema de gestión de la comunicación basado en la centralización y el control permitiría establecer y mantener el enfoque en el cliente. Schultz et al. (1994) animan a que los directivos mantengan el control de todos los 'puntos de contacto' posibles con la marca, es decir 
los puntos por los que el cliente está expuesto a la empresa. Siguiendo esta línea, Duncan y Caywood (1996) sugieren el uso de la investigación cuantitativa y cualitativa para cubrir todos los puntos de contacto del consumidor con la marca o la empresa, incluyendo tanto los contactos cuya iniciativa es del consumidor como los que tienen origen en la empresa:

De acuerdo con estas observaciones, es posible plantear las siguientes hipótesis:

Hla: El compromiso de la alta dirección influye positivamente en la implementación de programas de CIM.

H1b: La actitud de la alta dirección hacia el cambio influye positivamente en la implementación de programas de CIM.

H1c: La tolerancia al riesgo de la alta dirección influye positivamente en la implementación de programas de CIM.

FACTORES RELACIONADOS CON LA DINÁMICA INTER-DEPARTAMENTAL

Estos antecedentes pueden concretarse en:

a. Orientación al mercado y orientación a la marca. Algunas investigaciones han hallado una relación positiva entre la orientación al mercado y la orientación a la marca con la implementación de la CIM. A su vez, cada uno de estos factores afecta al otro de manera recíproca (Reid, 2005; Reid, Luxton y Mavondo, 2005).

b. Preparación, experiencia y habilidades. Moriarty (1993) afirma que las habilidades de gestión interdisciplinar constituyen la mayor barrera para la CIM. Por su parte, Beard (1996) manifiesta que los ejecutivos de las agencias y de las empresas-cliente no parecen confiar en la posibilidad de encontrar a personal con la preparación y experiencia necesaria como para trabajar en la CIM, siendo éste uno de los mayores obstáculos para su implantación exitosa.

c. Gestión interfuncional. Éste es a juicio de Duncan y Moriarty (1998) el factor organizativo más importante. La complejidad está en crear equipos interfuncionales que operen a nivel de valor de marca (brand equity) y equipos interfuncionales en el ámbito de la comunicación de marketing. En este sentido, Ambler y Barrow (1996) consideran que "las áreas separadas de Marketing y Recursos Humanos deben trabajar más juntas...un fuerte valor de marca con el cliente puede mejorar la rentabilidad de los RRHH, mientras que al mismo tiempo unos RRHH mejorados pueden mejorar la rentabilidad del valor de marca desde clientes externos". Este fenómeno sólo puede tener lugar si la alta dirección se compromete en destruir los muros entre los departamentos. La gestión interfuncional no sólo elimina las barreras existentes entre los departamentos y los grupos de interés (stakeholders), sino también ayuda a institucionalizar la retroalimentación, el feedback, y el aprendizaje. La comunicación debe ser gestionada como una actividad de ampliación de fronteras.

d. Los conflictos interdepartamentales y falta de comunicación horizontal. Entre los factores más mencionados en la literatura, por su influencia negativa en el desarrollo de la CIM se encuentran los conflictos interdepartamentales y la falta de comunicación horizontal. Sin una adecuada comunicación entre los departamentos las empresas pueden tener grandes dificultades en intercambiar y compartir información crucial acerca de los públicos objetivos (Pickton y Broderick, 2001). Kim et al. (2004) consideran que, en Estados Unidos, la 
tradición de una comunicación horizontal y una razonable competencia han ampliado el rango de elección del consumidor en una sociedad civil muy desarrollada y en un sistema capitalista. Todos estos factores han contribuido al origen de la CIM y a su desarrollo en este país.

e. La coordinación interfuncional. Según Schultz y Kitchen (2000), los procesos horizontales son necesarios para superar las barreras de especialización y estructuras jerárquicas que llevan a los profesionales de cada departamento hacia la alineación de sus actividades. Sólo a través de la alineación de los procesos de desarrollo estratégico, creación de valor, gestión de la información, etc., las empresas pueden tener una auténtica orientación al cliente.

De acuerdo con estas aportaciones, se formulan las siguientes hipótesis:

H2a: La orientación al mercado influye positivamente en la implementación de programas de CIM.

H2b: La orientación a la marca influye positivamente en la implementación de programas de CIM.

H2c: La preparación y la experiencia en gestión interfuncional influye positivamente en la implementación de programas de CIM.

H2d: La comunicación horizontal influye positivamente en la implementación de programas de CIM.

H2e: Los conflictos internos y el segmentalismo influyen negativamente en la implementación de programas de CIM.

H2f: El nivel de coordinación interfuncional influye positivamente en la implementación de programas de CIM.

H2g: La orientación al mercado y la orientación a la marca se influyen positivamente de forma recíproca.

\section{FACTORES RELACIONADOS CON LOS SISTEMAS ORGANIZATIVOS}

Al involucrar toda la empresa, la cuestión de cómo organizar el proceso de integración es la más crítica. Schultz (2000) concluye que los factores relacionados con el sistema organizativo representan los antecedentes que más influyen en la integración. La literatura de la CIM no sugiere sólo que la responsabilidad de la marca y la comunicación de marketing esté concentrada en una única localización central, sino que el contenido real de cada programa de comunicación esté organizado centralmente en un intento de asegurar la conformidad de los mensajes y evitar la dispersión de los recursos de comunicación (Schultz y Kitchen, 2000).

Es evidente que la comprensión del control de la comunicación es un asunto muy concreto. Duncan (1993) habla de "controlar e influir en todos los mensajes que los clientes y los stakeholders utilizan para formar una imagen y mantener una relación con una organización”. Sin embargo, este control central de los mensajes a menudo conlleva una frustración cuando los consumidores requieren respuestas para sus necesidades especiales o en circunstancias extraordinarias, como por ejemplo cuando un consumidor se encuentra con que se repiten unas respuestas grabadas o escritas según un modelo estándar para sus preguntas al ponerse en contacto con la empresa.

Como algunos autores indican, a menudo son las rígidas estructuras organizativas el principal impedimento para la integración (Christensen et al., 2008; Schultz y Schultz, 2003; Schultz 1999). Las tradicionales estructuras centralizadas 
basadas en el 'mando' y el 'control', deben ser sustituidas por modelos de respuesta rápida en las empresas de la nueva economía, y sólo cuando la gestión empieza a enfocarse en los resultados, en vez de en los beneficios, se contribuirá a la eliminación de los problemas de integración.

Beard (1996) sugiere que la estructura de la organización y los sistemas de compensación representan fuentes de conflicto y barreras para la implementación de CIM. Por su parte, Schultz et al. (1994) manifiestan que el uso de un enfoque basado en el consenso puede ser una solución con la que las empresas pueden superar estos conflictos internos. Asimismo, para Phelps, Harris y Johnson (1996) los asuntos que tiene que afrontar la comunicación de marketing son muy complejos y los múltiples entornos en que los mensajes de comunicación de marketing se desarrollan a menudo son turbulentos, de ahí que se requiera el aumento del uso del enfoque del consenso. Sin embargo, en muchas empresas, tres cuestiones lo obstaculizan: las recompensas individuales o por departamentos, el estilo de gestión inadecuado y el segmentalismo.

En primer lugar, las recompensas individuales crean problemas cuando un departamento de la empresa es juzgado sólo por su éxito en el corto plazo. La empresa crea un valor contraproducente con la realización individual, porque los reconocimientos se basan exclusivamente en respuestas rápidas en vez que en soluciones claras.

En segundo lugar, muchos estilos de gestión contrastan la puesta en marcha de un trabajo en equipo. Por un lado, la toma de decisión basada en el consenso lleva a una aparente pérdida de poder para el directivo, porque requiere que éste sea más que un líder. Según Phelps et al. (1996), tradicionalmente los directivos controlan los presupuestos, establecen los plazos, organizan y controlan. Los líderes desarrollan fuertes subordinados, animan el trabajo en grupo y en equipo y saben delegar el poder a los empleados (empowerment). Si la mayoría de los directivos defienden los principios de liderazgo, cuando hay presión por la necesidad de una decisión rápida, muchos directivos abandonan todas las premisas del modelo de consenso y adoptan el estilo de gestión "heroico". Los directivos tienden a indicar una voluntad de permitir que el grupo tome las decisiones, para luego proveer e imponer su propia decisión en vez de aceptar la posición del grupo. A pesar de 'salvar' temporalmente la situación, el estilo heroico destruye cualquier confianza en la toma decisión basada en el consenso por parte de los empleados (Phelps, et al., 1996).

Finalmente, incluso cuando los reconocimientos individuales y las acciones de gestión no impiden la toma de decisión basada en el consenso, las empresas fomentan el segmentalismo.

Los resultados del estudio realizado por Phelps et al. (1996) indican que a pesar de que la mayor parte de los encuestados todavía asignan la responsabilidad de desarrollar la estrategia de comunicación a una sola persona, el uso de un enfoque de consenso, donde los jefes de distintas funciones de comunicación trabajan conjuntamente para desarrollar estrategias de comunicación, se está empezando a expandir. Estos autores observan un aumento en la aplicación de la responsabilidad consensuada, en vez de la individual, para el desarrollo de la estrategia de comunicación de marketing, pero no muestran específicamente el grado en que las empresas están implementando la CIM.

De todo lo anterior se pueden plantear las siguientes hipótesis de investigación: 
H3a: La división de la organización en un alto número de departamentos, llamada departamentalización, influye negativamente en la implementación de programas de integración.

H3b: Una estructura centralizada de la empresa influye negativamente en la implementación de programas de CIM.

H3c: El modelo de toma de decisiones basado en el consenso influye positivamente en la implementación de programas de CIM.

H3d: Los sistemas de compensación individuales influyen negativamente en la implementación de programas de CIM.

\section{OTROS FACTORES ANTECEDENTES}

Entre otras variables que destacan en la literatura como antecedentes internos de la CIM, encontramos el tamaño de la organización y la tipología de empresa.

a. Tamaño de la empresa. Algunos autores sugieren que el tamaño de una organización también puede influir en la habilidad de ésta para implementar la CIM (Cornelissen, Lock y Garner, 2001; Low, 2000; Nowak y Phelps, 1994; Reid, 2005). Concretamente, Low (2000) realizó un estudio empírico en el que concluyó que empresas más pequeñas con jerarquías de marca menos complejas eran menos susceptibles de aplicar variados programas de comunicación de marketing, adoptar una rígida formalización departamental $\mathrm{y}$, por consiguiente, eran más susceptibles de ser integradas o de adoptar procesos más coherentes con la CIM. También Cornelissen, Lock y Garner (2001); Nowak y Phelps (1994) encontraron una relación negativa entre el tamaño de la empresa y el nivel de implementación de la CIM.

b. Con respecto a la tipología de empresa, Low (2000) sugiere que las empresas de servicios son más propensas a ser más integradas con respecto a las empresas centradas en productos, basándose en la premisa según la cual las empresas de servicios tienen un mayor acceso a los clientes. Por el contrario, Reid (2005) identificó empíricamente que es más probable que la aplicación de la CIM tenga lugar en grandes empresas manufactureras que en más pequeñas empresas de servicios, puesto que empresas más grandes harán más esfuerzos de cara a mejorar los procesos de planificación, tener mecanismos más formales de recogida y uso de datos sobre el consumidor, y pueden gestionar y coordinar un número mayor de grupos interfuncionales $\mathrm{y}$ agencias externas. Este autor comprobó empíricamente que el proceso de la CIM prevalece en las empresas más grandes o las que son líderes de su mercado y en empresas que cuentan con grandes presupuestos.

De acuerdo al debate anterior se plantean las siguientes hipótesis:

H4a: El tamaño de la organización influye positivamente en la implementación de programas de integración.

H4b: La posición de liderazgo de la organización con respecto a la competencia influye positivamente en la implementación de programas de CIM. 


\section{H4c: La tipología de empresa influye significativamente en la}

implementación de programas de CIM.

\subsubsection{Antecedentes exógenos}

Entre los factores externos que impulsan u obstaculizan el desarrollo de la CIM, destacamos los siguientes: el nivel de competencia, la turbulencia tecnológica, y el entorno cultural e institucional en el que actúa la empresa.

\section{EL NIVEL DE COMPETENCIA}

Un entorno de mercado hostil caracterizado por una intensa competencia y entornos dinámicos caracterizados por rápidos avances tecnológicos y cambios continuos en las preferencias de los consumidores, influyen de manera significativa en los resultados de las empresas (Low, 2000; Reid, 2005). Reid (2005) confirma en su trabajo empírico que la turbulencia del mercado y el alto nivel de competencia tienen una relación positiva con el nivel de CIM logrado. El deseo de ser competitivo en estas condiciones ambientales, pueden dar el impulso para que las empresas implementen la CIM de cara a facilitar la coordinación estratégica de los mensajes de marca y el efecto de la estrategia de marca.

Este resultado coincide con los del estudio de Low (2000), que indica que la intensidad competitiva influye positivamente en el grado de aplicación de la CIM. Las organizaciones que compiten en mercados caracterizados por una intensa y turbulenta competencia pueden encontrar beneficios en la integración estratégica de la comunicación de marca para maximizar los efectos de las actividades de comunicación. Además, el desarrollo de experiencia en la planificación e implementación de CIM contribuye a mejorar la habilidad de competir y responder a los esfuerzos de marketing de los competidores.

\section{LA TURBULENCIA TECNOLÓGICA}

Según Schultz (1996) un elemento que lleva a la necesidad de enfoques integrados es la rapidez de evolución de la tecnología de la información. Pero según este autor no se trata sólo de tecnología de la comunicación, sino de toda la revolución tecnológica que nos está trasladando desde la revolución industrial hacia la revolución de la información. La tecnología está creando la intersección entre comunicación y distribución, reduciendo el mundo en un amplio mercado interconectado y globalizado. La irreversibilidad de la tecnología conlleva la necesidad y demanda de integración.

\section{EL ENTORNO CULTUAL}

Según Kim et al. (2004) la CIM será aceptada como nuevo paradigma del marketing si el entorno del mercado se mueve para promover la CIM. Sin embargo, algunas circunstancias sociales e institucionales en algunos países pueden ser importantes factores para impulsar u obstaculizar el desarrollo de la CIM. Dentro del marco de estas observaciones, Kim et al. (2004) proponen un "modelo de la tasa de desarrollo" de CIM según el cual el proceso de CIM se describe como altamente dinámico, donde el mercado y el entorno del marketing representan las fuerzas que impulsan la implementación de la CIM. Los autores explican como el nivel de coordinación cambia dependiendo de la medida en que el denominador común de intereses se forma entre clientes y agencia de publicidad junto con la segunda y tercera agencias del contexto. El tipo de coordinación e integración variará más porque los sectores tendrán distintas relaciones el uno con el otro en términos de niveles de interés. Si algunos factores actúan a favor de la CIM y otros son contrarios, entonces se crearían 
situaciones dialécticas. A pesar de que la fuerza de las barreras puede ser limitada gracias a las ventajas económicas de la CIM, no es posible predecir con certeza los factores dominantes en cada proceso.

Siguiendo estos planteamientos, se formulan las siguientes hipótesis:

H5a: El nivel de competencia del mercado en que opera la organización influye positivamente en la implementación de programas de integración.

H5b: La turbulencia tecnológica del entorno influye positivamente en la implementación de programas de CIM.

H5c: El entorno cultural e institucional en el que actúa la empresa influye significativamente en la implementación de programas de CIM.

\subsection{Consecuentes de la CIM}

En este apartado se pretende analizar y clasificar las consecuencias que derivan de la aplicación del paradigma de CIM y revisar las principales conclusiones alcanzadas en los estudios realizados al efecto.

Siguiendo a diversos autores (Duncan y Moriarty, 1998, 2004; Kitchen y Schultz, 1999; Reid, 2003, 2005; Swain, 2004; Reid, Luxton y Mavondo, 2005) se distinguen tres categorías de consecuentes de la implementación de la CIM, siguiendo su evolución desde una herramienta meramente táctica hasta llegar a ser un paradigma estratégico.

\subsubsection{Resultados relacionados con la performance de la comunicación de marketing}

Los resultados del estudio de Low (2000) indican que la efectividad de la publicidad, la promoción de ventas, el marketing directo y las relaciones públicas es mayor cuando hay un nivel alto de CIM, corroborándose pues una fuerte relación entre la CIM y la performance de la comunicación de marketing.

Por la escasa evidencia empírica sobre la relación entre CIM y los distintos resultados de la organización, es difícil especificar una exacta relación entre ellos. Sin embargo, desde una perspectiva operativa, se puede suponer que la CIM es susceptible de proveer beneficios en la coordinación de las actividades de comunicación de marketing y entre las distintas funciones que se ven involucradas en la implementación de campañas de marketing (Beard, 1996; Cornelissen y Lock, 2000; Schultz, 1993; Stewart, 1996).

Cornelissen y Lock (2000) presentan estos beneficios como psicológicos e incluyen una reducción del conflicto y una disminución de costes de transacción entre las funciones. La CIM ofrece ahorro en costes adicionales relacionados con la infraestructura organizativa, en la que la cooperación entre los departamentos de la organización evita la innecesaria duplicación de estrategias de comunicación, llevando de esta manera a una mejora en la eficiencia operativa y en la coherencia del mensaje. Desde una perspectiva de campañas individuales, sería de esperar que las firmas que han implementado con éxito la CIM experimenten un aumento de los ingresos de cada campaña, resultados funcionales, entre los que se incluyen la sinergia entre los elementos del mix de comunicación de la campaña y la mejora de la capacidad de emplear un rango más amplio y apropiado de herramientas (Duncan y Moriarty, 1997). 
El éxito de campañas puede medirse potencialmente utilizando un análisis económico de la rentabilidad de la inversión a través de los objetivos directos de comportamiento y, en una base más subjetiva, sobre las percepciones de directivos acerca del éxito relativo a campañas de la competencia. En general, es de esperar que las organizaciones que empleen la CIM tengan una mayor capacidad de alcanzar sus objetivos directos e indirectos de campaña establecidos, como un aumento del conocimiento y preferencia de la marca, de la actitud hacia la marca y de la intención de compra.

Duncan y Mulhern (2004) a su vez destacan que las organizaciones que implementen mejor la CIM tendrán una mayor capacidad de lograr los objetivos de campaña directos e indirectos prefijados.

Teniendo en cuenta todo lo anterior se formulan las siguientes hipótesis:

H6a: El nivel de aplicación de la CIM influye positivamente en la performance de la comunicación de marketing.

H6b: El nivel de aplicación de la CIM influye positivamente en la consecución de los objetivos de campaña (directos e indirectos).

H6c: El nivel de aplicación de la CIM influye positivamente en la eficiencia operativa y en la reducción de los conflictos internos.

H6d: La performance de la comunicación de marketing influye positivamente en la performance de marca.

\subsubsection{Resultados relacionados con la performance de la marca}

En general, se observa cierto acuerdo entre los investigadores sobre la correlación que existe entre la aplicación del proceso de CIM y los resultados empresariales.

Kitchen et al. (2004) ponen de manifiesto que la CIM ya no representa sólo un proceso de comunicación, sino un proceso asociado con la gestión de las marcas. Además, según estos autores, la CIM involucra la gestión de comunicación de marketing de una manera más exhaustiva y holística para lograr objetivos estratégicos.

Por su parte, Reid, Luxton y Mavondo (2005) proponen la medida de los resultados de marca a dos niveles interrelacionados, incluyendo la performance de la comunicación de marketing y la performance de marca. Uno de los efectos más deseables de una CIM eficaz es la consecución de una ventaja competitiva en el mercado (Rust et al., 2004). Swain (2004) precisa que entre los efectos deberían incluirse un conjunto de resultados relacionados con la marca y con el cliente: su notoriedad, un aumento de la actitud positiva hacia la misma, una mayor preferencia de la marca, una mayor intención de compra con respecto a la marca y la facilitación de la compra.

Low (2000) realizó una de las primeras investigaciones que apoyan la relación entre CIM y estos resultados de marca, pero el mismo autor observa que se obtienen resultados difíciles de explicar que ponen de manifiesto la necesidad de una más exhaustiva conceptualización y definición de la CIM, así como del desarrollo de herramientas más fuertes para la medición de la misma. Recientemente Zahay et al. (2004) han abordado el análisis de un rango de relaciones con los clientes y medidas de la marca para estudiar su relación con la implementación de la CIM.

Según estas aportaciones, cabe plantear las siguientes hipótesis: 
H7a: El nivel de aplicación de la CIM influye positivamente en la notoriedad de la marca.

H7b: El nivel de aplicación de la CIM influye positivamente en la actitud positiva hacia la marca.

H7c: El nivel de aplicación de la CIM influye positivamente en la preferencia de la marca.

H7d: El nivel de aplicación de la CIM influye positivamente en la intención de acción de compra con respecto a la marca.

\subsubsection{Resultados relacionados con el cliente y otros stakeholders}

Schultz y Schultz (1998) introducen como medida de la implementación de la CIM la medición de la rentabilidad de la inversión en el cliente (ROCI, return on customer investment). A su juicio, la atención ya no se centra en intentar medir el efecto de los esfuerzos de comunicación individuales como respuesta a una publicidad determinada o a un evento especial, al ser imposible en este contexto aislar los efectos de los esfuerzos individuales. Al contrario, la atención se debe centrar en relacionar la inversión realizada en un cliente específico o en un grupo bien definido de clientes con el aumento (o la disminución) de los flujos de ingresos procedentes de estos mismos clientes. Una vez que las organizaciones hayan puesto en marcha los procesos y las infraestructuras necesarias para medir atentamente la rentabilidad de la inversión a un nivel general, éstas pueden testar diferentes mix de comunicación o niveles de inversión para posteriormente ajustar el plan para ciclos futuros. El punto más importante es que, en este enfoque, los flujos de ingresos del cliente representen el elemento crítico de medición y no los esfuerzos individuales de comunicación.

En una reciente publicación, Rust et al. (2004) desarrollaron un marco teórico que conecta la estrategia y las tácticas de marketing con los beneficios de clientes, del mercado y financieros. Desde una perspectiva de CIM, este autor identifica el impacto de la estrategia y tácticas de marketing sobre las actitudes, la lealtad, la satisfacción y la retención del cliente. Dichas medidas intermedias de la performance pueden ser agregadas a nivel de marketing y medidas a través de escalas relacionadas con el valor de marca y valor de cliente. Finalmente estas acciones surten efectos sobre el impacto financiero, medido por la posición financiera que incluye los beneficios y el flujo de caja. El resultado de esta cadena de impactos se nota en el aumento del valor de la organización y del mercado de la marca.

Ambler, Bhattacharya, Edell, Keller, Lemon y Mittal (2002) coinciden con Kitchen y Schultz (2001) en utilizar la ROI (rentabilidad de la inversión), mientras que otros autores prefieren la ROTPI (return on touch point investment), es decir la medida de la rentabilidad de la inversión en el punto de contacto con el cliente (Schultz, Cole y Bailey, 2004) y la estimación de las mejoras en el valor de la marca y en el valor del cliente (Duncan y Mulhern, 2004; Hutton, 1996).

Reid, Luxton y Mavondo (2005) favorecen la existencia de una 'cadena de productividad de la CIM' que conecte la performance de la gestión de comunicación de marketing y de campañas con los resultados relacionados con los clientes y el valor de marca.

Desde una perspectiva de relación con el cliente, Duncan y Moriarty (1997) y Duncan y Mulhern (2004) auspician el desarrollo de una serie de medidas, como la 
rentabilidad del punto de contacto con el cliente ("return on customer touch point"), la rentabilidad del cliente, el valor del cliente a lo largo de su 'ciclo de vida', listados de novedad y referencia, ratios de migración del cliente, y cuotas de clientes.

En un sentido más amplio, los resultados basados en el mercado que proceden de la CIM pueden estar relacionados con el concepto de la cadena de valor de la marca (Rust et al., 2004), que identifica una cadena de inversiones en marketing y su potencial impacto en la mente del consumidor, en la performance de mercado y el valor para el accionista. Más recientemente, Beard $(1996 ; 1997)$ publicó un estudio empírico del que se deduce que la aplicación de la CIM está relacionada con una percepción de resultados positivos para las empresas-cliente en sus relaciones con las agencias externas de comunicación.

Una explicación de estos resultados deriva de los beneficios del paradigma de la CIM, que enfatiza la igualdad y la pertenencia a los objetivos comunes que las funciones $\mathrm{y}$ los especialistas de comunicación de marketing comparten. Las características de la CIM deberían facilitar la integración y la coordinación entre las disciplinas y los profesionales de comunicación de marketing, de ahí que los directivos de comunicación y de marketing que utilizan la CIM podrán encontrar más fácil la labor de gestión de una o más agencias externas y las relaciones entre las mismas. Esta conclusión sería coherente con los resultados del estudio de Kitchen y Schultz (1997), en el que los sondeos a ejecutivos de agencias de publicidad del Reino Unido revelan que los encuestados creen que los programas de CIM en una agencia pueden ayudar a integrar los esfuerzos del cliente de esta agencia. Otra explicación puede residir en que la implementación de la CIM no lleva necesariamente a una disminución de la función de la publicidad, tal y como sugieren Phelps y Johnson (1996, p.13), que consideran que el aumento de responsabilidad de una función de comunicación de marketing no coincide con la disminución de la responsabilidad de otra u otras funciones.

Según estas aportaciones, concluimos que:

H8a: El nivel de aplicación de la CIM influye positivamente en las actitudes del cliente.

H8b: El nivel de aplicación de la CIM influye positivamente en la lealtad del cliente.

H8c: El nivel de aplicación de la CIM influye positivamente en la retención del cliente.

H8d: El nivel de aplicación de la CIM influye positivamente en las relaciones de la empresa con las agencias externas.

H9a: Los resultados de comunicación de marketing influyen positivamente en los resultados financieros.

H9b: Los resultados de marca influyen positivamente en los resultados financieros.

H9c: Los resultados a nivel de clientes influyen positivamente en los resultados financieros.

H9d: Los resultados a nivel de relación entre la empresa-cliente y las agencias externas influyen positivamente en los resultados de la empresa. 


\subsection{Modelo teórico de antecedentes y consecuentes de la CIM}

Tras la amplia revisión de la literatura realizada en los apartados anteriores, se propone un modelo teórico de funcionamiento de la CIM (figura 1) que trata de recoger de una manera exhaustiva todas aquellas variables que de una manera positiva o negativa pueden afectar al grado de integración alcanzado por una organización.

Asimismo, como consecuencia de alcanzar el deseado grado de integración se producirán determinados efectos sobre la eficacia de los esfuerzos de comunicación de marketing, sobre el valor de marca, del cliente y los resultados financieros de la organización en su conjunto.

Este modelo conceptual propuesto abre el camino para que la investigación académica futura aborde trabajos empíricos que conduzcan a testar de manera global o en parte las hipótesis teóricas establecidas. Buena parte de las medidas requeridas han sido ya desarrolladas y ampliamente validadas por la investigación académica previa. Conceptos como la orientación al mercado, orientación a la marca, turbulencia tecnológica, valor de marca, valor del cliente, o eficacia de la comunicación de marketing han sido ampliamente utilizados por los investigadores académicos y no resulta especialmente problemática su medida.

Sin lugar a dudas, la parte más difícil del modelo es la que tiene que vez con el desarrollo y validación de escalas que sean capaces de capturar la esencia de la CIM. Como punto de partida, el instrumento de medida de Duncan y Moriarty (1997), miniauditoría de comunicación integrada de marketing, puede resultar extremadamente útil para testar esas relaciones.

La técnica de análisis de datos más apropiada para testar el modelo propuesto, por las relaciones causales que plantea, son los modelos de ecuaciones estructurales, bien en su vertiente de modelos basados en la covarianza (Lisrel, Amos, EQS) o en la varianza (PLS).

En definitiva, la estimación del modelo propuesto proporcionará a los investigadores académicos una mejor comprensión de lo que representa la comunicación integrada de marketing dentro de la gestión empresarial contemporánea, las principales variables que la determinan o frenan, así como los principales beneficios que su implementación produce. 


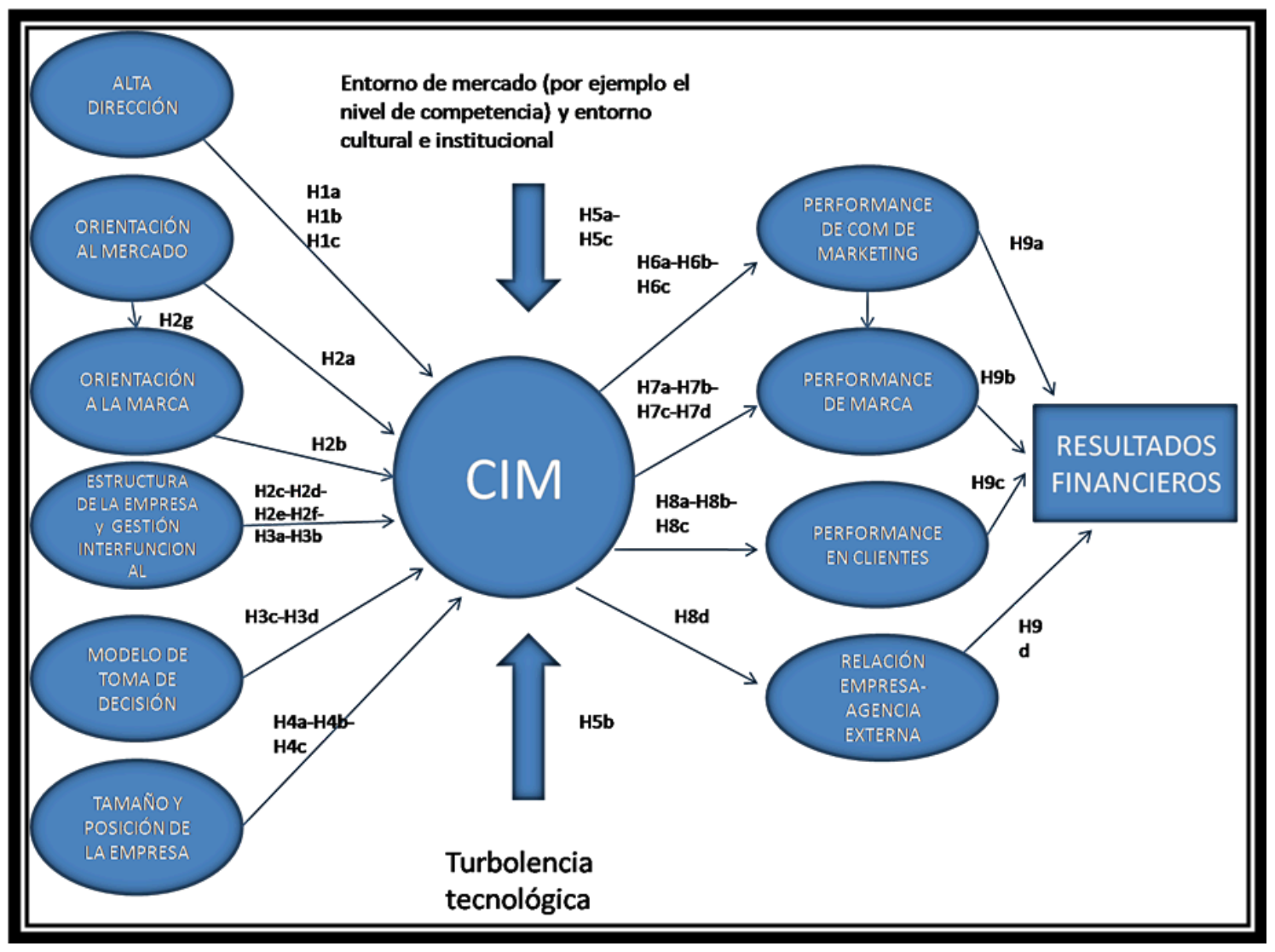

Fuente: Elaboración Propia

\section{CONCLUSIONES}

Este trabajo lleva a cabo una importante reflexión teórica acerca del concepto de la CIM, sus antecedentes y consecuentes, que nos conducen a la propuesta de una definición propia y al planteamiento de un modelo teórico de antecedentes $\mathrm{y}$ consecuentes de la CIM, siguiendo las hipótesis previamente formuladas tras la una exhaustiva revisión de la literatura.

El análisis de las conceptualizaciones de la CIM desarrolladas desde la introducción del concepto hasta la actualidad, refleja su evolución desde una herramienta meramente táctica hasta llegar a representar un importante elemento estratégico que interesa a toda la organización y no sólo el área de la comunicación de marketing. A lo largo de la evolución del concepto, se asiste a un cambio de enfoque: se pasa de la perspectiva inside-out a la visión outside-in, centrada en el cliente. En definitiva, la CIM representa una importante ventaja competitiva sólo si representa una componente estratégica que guía todas las actuaciones de la empresa. En este trabajo se propone una definición original de la CIM que representa la base teórica imprescindible para la propuesta de un modelo de desarrollo de la misma como proceso interactivo.

En primer lugar, en el modelo se destaca un conjunto de factores internos, que incluyen los aspectos relacionados con la alta dirección, la orientación al mercado, la orientación a la marca, la estructura de la empresa, el modelo de toma de decisión, y la gestión 
interfuncional. Además, el tamaño de la organización y el sector de actividad representan otros antecedentes de carácter interno.

En segundo lugar, se identifican algunos antecedentes externos que incluyen la turbulencia tecnológica, el nivel de competencia del sector y el entorno cultural e institucional en el que opera la empresa.

El primer factor 'alta dirección', incluye tres aspectos: el compromiso de la alta dirección con la aplicación de la CIM, su actitud al cambio y su tolerancia al riesgo. Todos estos aspectos parecen influir positivamente en el grado de implementación de la CIM. De la misma manera la orientación al mercado y la orientación a la marca representan dos factores interrelacionados que se influyen recíprocamente y que, a su vez, tienen una influencia positiva en la CIM.

Dentro de la estructura de la empresa y la gestión interfuncional se engloba un conjunto de aspectos que ejercen una influencia negativa en la aplicación de esta nueva filosofía: la estructura centralizada de la empresa, la división en un alto número de departamentos, los conflictos internos y el consecuente segmentalismo. En cambio, otros aspectos, como el nivel de coordinación interfuncional y de comunicación horizontal, influyen positivamente en su implementación.

Respecto al modelo de toma de decisiones, se observa un acuerdo unánime de los investigadores en considerar que un modelo de decisión basado en el consenso representa un factor que influye positivamente. Sin embargo, los sistemas de remuneración individuales obstaculizan el desarrollo de la deseada integración.

Otros aspectos internos influyen positivamente en la CIM como el tamaño de la empresa y su posición con respecto a la competencia. En la literatura no parece clara la influencia que ejerce el sector de actividad de la organización en la aplicación del paradigma de CIM.

Finalmente, se destacan algunos antecedentes externos que influyen positivamente como la turbulencia tecnológica, la intensidad competitiva y el entorno cultural e institucional en el que la empresa se encuentra a operar.

Los consecuentes de la CIM se recogen en tres categorías: los efectos relacionados con la performance de comunicación de marketing, los resultados relacionados con la performance de marca y finalmente los efectos relacionados con el cliente y otros stakeholders.

En primer lugar, cabe destacar que la CIM contribuye a la consecución de los objetivos de campaña, directos e indirectos, a la reducción de los conflictos internos y a la eficiencia operativa de la comunicación de marketing. Todos estos efectos, relacionados con la performance de comunicación de marketing, influyen positivamente en la performance de marca. En segundo lugar, la implementación de la CIM influye positivamente en la performance de marca, es decir en su notoriedad, en su preferencia, en la actitud positiva hacia la marca y en la intención de compra con respecto a la marca. Estos efectos se recogen en la segunda categoría de consecuentes. En tercer lugar, el grado de aplicación de la CIM influye positivamente en las actitudes del cliente, en su lealtad, en la retención de los clientes y en las relaciones de la empresa con las agencias externas. Finalmente, la consecución de los efectos que se acaban de detallar influye positivamente en los resultados financieros de la empresa.

Conseguir la operatividad de este modelo, además de respaldar los efectos en los stakeholders y en la performance de la empresa, permitirá aclarar la relación de la CIM 
con otros conceptos de marketing, como la orientación al mercado y la orientación a la marca. La parte más difícil de este modelo está relacionada con el desarrollo de escalas que capten adecuadamente la esencia del proceso de integración de la comunicación de marketing que puedan llevar a medidas adecuadas de su performance externa. El uso de instrumentos existentes, como el miniaudit de Duncan y Moriarty (1997), puede representar un punto de partida aceptable pero no suficiente.

En definitiva, una de las principales barreras para el desarrollo de la CIM sigue siendo la dificultad en la elaboración de herramientas de medida del grado de implementación de CIM del que derivan los efectos que numerosos autores han mencionado en sus publicaciones y que encuentran respaldo en este artículo. Sin embargo, sin una adecuada medición del concepto de CIM es imposible llegar a la medición de los resultados que la aplicación de este paradigma conlleva. 


\section{Bibliografía}

Ambler, T.; Barrow, S. (1996), “The employer Brand”, Journal of Brand Management, Vol. 4, N.3, pp.185-206.

Ambler, Tim, C. B. Bhattacharya, Julie Edell, Kevin Lane Keller, Katherine N. Lemon, and Vikas Mittal (2002), "Relating Brand and Customer Perspectives on Marketing Management", Journal of Service Research, 5 (August), 13-25.

Beard, F. (1996), "Integrated Marketing Communications: New Role Expectations and Performance Issues in the Client-Ad Agency Relationship?", Journal of Business Research, Vol. 37, N.3, pp. 207-215.

Bigné, E. (2001), Promoción Comercial, Esic Editorial, Madrid.

Borden, N. H.(1964), "The Concept of the Marketing Mix", Journal of Advertising Research, Vol. 4, pp.2-7.

Caywood, C.; Schultz, D. E.; Wang, P. (1991), Integrated Marketing Communications: a survey of National Goods Advertisers, informe no publicado, Bloomington, IN: Medill School of Journalism, Northwestern University, June.

Christensen, L. T.; Firat, A. F.; Torp, S. (2008), "The organization of integrated communications: toward flexible integration", European Journal of Marketing, Vol. 42, n. $3 / 4$, pp. $423-452$.

Cornelissen, J. P (2001), "Integrated marketing communications and the language of marketing development”, Journal of Marketing Communications, Vol. 20, pp.483-498.

Duncan, T.; Caywood, C. (1996), "The concept, process, and evolution of Integrated Marketing Communication", en Thorson, E.; Moore, J., Integrated Communication: Synergy of Persuasive Voices, Mahwah, NJ, Lawrence Erlbaum Associates, pp. 13-34.

Duncan, T. (2005), Principles of Advertising \& IMC, 2nd ed., McGraw-Hill, Boston, MA.

Duncan, T. (2002), IMC: Using Advertising and Promotion to Build Brands (International Edition), New York, The Mc Graw-Hill Companies, Inc.

Duncan, T.; Everett, S. E. (1993), "Client Perceptions of Integrated Communications", Journal of Advertising Research, Vol.32, N.3, pp. 30-39.

Duncan, T.; Moriarty, S. E. (1997), Driving Brand Value, New York, McGraw Hill.

Duncan, T.; Moriarty, S. E. (1998), “A Communication-Based Marketing Model for Managing Relationships", Journal of Marketing, Vol.62, April, pp.1-13.

Duncan, T.; Mulhern, F. (2004), A White paper on the status, scope and future of IMC, New York, McGraw Hill.

Eagle, L.; Kitchen, P. J. (2000), "IMC, brand communications, and corporate cultures: client/advertising agency co-ordination and cohesion", European Journal of Marketing, Vol. 34, N. 5/6, pp. 667-686.

Fill, C. (2002), Marketing Communications: Contexts, Strategies and Applications, 3rd ed. London, Europe, Prentice Hall Limited.

Gould, S. J. (2004), "IMC as Theory and as Poststructural Set of Practices and Discourses: A Continuously Evolving Paradigm Shift", Journal of Advertising Research, Vol.44, March, pp.66-70. 
Grönroos, C. (1996), "Relationship marketing logic", Australian Marketing Journal, N.1.

Gronstedt, A. (1996), "How agencies can support Integrated Marketing Communications", Journal of Business Research, Vol. 37, pp. 201-206.

Hutton, J. G. (1996), "Integrated Marketing Communications and Evolution of Marketing Thought", Journal of Business Research, Vol. 37, pp.139-146.

Kim, I.; Han, D.; Schultz, D. E. (2004), "Understanding the Diffusion of Integrated Marketing Communication", Journal of Advertising Research, Vol.44, March, pp.3145 .

Kitchen, P. J.; Brignell, J.; Li, T.; Spickett-Jones, G. (2004), "The Emergence of IMC: A Theoretical Perspective", Journal of Advertising Research, Vol.44, March, pp.19-30.

Kitchen, P. J.; Schultz, D.E. (1997), "Integrated Marketing Communications in US Advertising Agencies: An exploratory Study", Journal of Advertising Research, Vol.37, N.5, pp.7-18.

Kitchen, P.J.; Schultz, D. E. (1999), "A multi-country comparison of the drive for IMC", Journal of Advertising Research, Vol. 39, N.1, pp. 21-38.

Kitchen, P.J.; Schultz, D. E. (2000), “A response to 'Theoretical Concept or Management Fashion", Journal of Advertising Research, Vol. 40, N.5, pp.17-21.

Kotler, P. (2000), Marketing Management, 10th edition London, Prentice Hall International Limited, UK.

Lagioni (2004), CIM Comunicazione di Marketing Integrata, Una nuova cultura della comunicazione d'impresa, Ed. Tecniche Nuove, Milano.

Lee, D. H.; Park, C. W. (2007), "Conceptualization and Measurement of Multidimensionality of Integrated Marketing Communications", Journal of Advertising Research, Vol. 47, September, pp. 222-236.

Low, G. L. (2000), “Correlates of Integrated Marketing Communications", Journal of Advertising Research, Vol.40, N.3, pp. 27-39.

Moriarty, S.E. (1993) "The circle of synergy: theoretical perspectives and an evolving IMC research agenda", en Thorson E.; Moore, J., Integrated Communication: Synergy of Persuasive Voices, Mahwah, NJ, Lawrence Erlbaum Associates, pp. 333-54.

Nowak, G.; Phelps, J. (1994), “The integrated Marketing Communications Phenomenon: An examination of its impact on Advertising practices and its Implications for Advertising Research", Journal of Current Issues and Research in Advertising, Vol. 16, N.1, pp.49-66.

Peppers, D.; Rogers, M. (2001), Impresa One to One, Il Marketing Relazionale nell'era della rete, Edizione Apogeo.

Percy, L.; Rossiter, J. R.; Elliot, R. (2001), Strategic Advertising Management, New York, Oxford University Press Inc. (en Kim, I.; Han, D.; Schultz, D. E. (2004), "Understanding the Diffusion of Integrated Marketing Communication", Journal of Advertising Research, Vol.44, March, pp.31-45).

Phelps, J. E.; Johnson, E. (1996), "Entering the quagmire: examining the 'meaning' of integrated marketing communications", Journal of Marketing Communications, Vol. 2, N. 3, pp.159-172. 
Phelps, J.; Harris, T.E.; Johnson, E. (1996), "Exploring decision-making approaches and responsibility for developing marketing communications strategy", Journal of Business research, Vol. 37, N. 3, pp. 217-223.

Pickton, D.; Broderick, A. (2001), Integrated Marketing Communications, PrenticeHall, Hemel Hempstead.

Pickton, D.; Harley, B. (1998), "Measuring Integration: an assessment of the quality of integrated marketing communications", International Journal of Advertising, Vol. 17, pp. 447-457.

Rapp, S.; Collins, T. (1988), Maximarketing: the New Direction in Advertising, Promotion and Marketing Strategy, New York: NAL Penguin Books.

Rapp, S.; Collins, T. (1990), The Great Marketing Turnaround: the Age of the Individual and How to Profit from It, Englewood Cliffs, NJ, Prentice Hall.

Roman, E. (1988), Integrated Direct Marketing: Techniques and Strategies for Success, New York, McGraw-Hill Book Company.

Reid, M. (2005), "Performance Auditing of Integrated Communication (IMC) actions and outcomes", Journal of Advertising, Vol. 34, N.4, pp.41-54.

Reid, M.; Luxton, S.; Mavondo, F. (2005), "The relationship between Integrated Marketing Communication, Market Orientation and Brand orientation", Journal of Advertising, Vol. 34, N. 4, pp. 11-23.

Rossiter, J. R.; Bellman, S. (2005), Marketing Communications: Theory and Applications, Victoria, Pearson Prentice Hall, Australia.

Rust, R. T.; Ambler, T.; Carpenter, G. S.; Kumar, V.; Srivastava , R. K. (2004), "Measuring Marketing Productivity: Current Knowledge and Future Directions", Journal of Marketing, Vol. 68, Octubre, pp. 76-89.

(1996), "The inevitability of Integrated Communications", Journal of Business Research, Vol. 37, pp.139-146.

Schultz, D.E.; Cole, B.; Bailey, S. (2004), "Implementing the 'Connect the Dots' Approach to Marketing Communication”, International Journal of Advertising, N. 23, pp. 455-477.

Schultz, D.E.; Kitchen, P. J. (2000), Communicating Globally, An Integrated Marketing Approach, Edition NTC Business Books.

Schultz, D.E.; Kitchen, P. J. (1997), "Integrated marketing communications in U.S. advertising agencies: an exploratory study", Journal of Advertising Research, Vol. 37, N. 5, pp. 7-18.

Schultz, D. E.; Schultz, H. F. (1998), "Transitioning marketing communication into the twenty-first century", Journal of Marketing Communications, Vol. 4, N. 1, pp. 9-26.

Schultz, Tannenbaumm y Lauterborn (1994), The new Marketing Paradigm. Integrated Marketing Communication, NTC Business Books, Chicago, IL.

Shimp, T.A. (2003), Advertising, Promotion and Supplemental Aspects of Integrated Marketing Communications, 6th ed., Thomson South-Western, Cincinnati, OH.

Smith, P. (1996), "Benefits and Barriers to Integrated Communications", Admap, February, pp.19-22, en Reid, M. (2005), "Performance Auditing of Integrated 
Communication (IMC) actions and outcomes", Journal of Advertising, Vol. 34, N.4, pp.41-54.

Stephens, D. L.; Hill, R.P.; Bergman (1996), "Enhancing the Consumer-Product Relationship: Lessons from the QVC Home Shopping Channel”, Journal of Business Research, pp.193-200.

Stevenson, B. (1989), "Critical marketing strategies for the 1990s", Journal of Direct Marketing, Vol. 3, N. 3, pp. 34-41.

Stewart, D. W. (1996), "Market-Back Approach to the Design of Integrated Marketing Communications Programs: A change in Paradigm and a Focus on Determinants of Success", Journal of Business Research, Vol. 37, pp.159-172.

Swain, W. N. (2004), "Perceptions of IMC after a Decade of Development: Who's at the Wheel, and How Can We Measure Success?", Journal of Advertising Research, Vol. 44, N. 1, pp.46-65.

Zahay, D.; Peltier, J.; Schultz, D. E.; Griffin, A. (2004), "The Role of Transactional versus Relational Data in IMC Programs: Bringing Customer Data Together", Journal of Advertising Research, Vol. 44, N. 1, pp.46-65. 


\title{
Una propuesta multidimensional para la medición de la comunicación integrada de marketing (CIM)
}

\author{
Lucía Porcu \\ Licenciada en Relaciones Públicas por la Università degli Studi di Udine, Italia \\ lusiettados@gmail.com \\ Salvador del Barrio García \\ Doctor en Ciencias Económicas y Empresariales por la Universidad de Granada \\ dbarrio@ugr.es
}

Facultad de Ciencias Económicas y Empresariales

Universidad de Granada

Campus de Cartuja s/n

18071 Granada

Telf. 958242347

\section{Resumen}

Desde que en la década de los 80 las grandes agencias de publicidad norteamericanas comenzaran a comprender la necesidad de la integración del mix promocional como fuente de ventaja competitiva el interés por la comunicación integrada de marketing (CIM) ha ido creciendo tanto en la industria de la publicidad y comunicación como en la investigación académica. Tras un amplio debate, aun inacabado, acerca de lo que representa y significa la CIM, en los últimos años la investigación académica se ha centrado en proponer una medida del grado de integración alcanzado en las organizaciones y sus efectos sobre otras variables relacionadas con la performance del mercado (imagen corporativa, valor de marca, rentabilidad, etc.). En este trabajo se exponen las herramientas de medición más destacadas hasta la fecha y se propone una nueva escala basada en la CIM como concepto multidimensional.

Palabras clave: Comunicación Integrada de Marketing, CIM, escala de medida 


\section{Introducción}

La comunicación de masas ya no funciona. Con esta afirmación Schultz, Tannenbaum y Lauterborn (1993) introdujeron el concepto Integrated Marketing Communications como alternativa a la concepción tradicional de la comunicación de marketing centrada en torno a la publicidad. En Estados Unidos hace varias décadas el $75 \%$ de los presupuestos de marketing iban destinados a la publicidad, mientras que en la actualidad ese porcentaje se ha reducido al $25 \%$, lo que refleja un cambio en la forma de entender el papel que juega la comunicación en las organizaciones modernas.

En la década de los 90 se comienza a plantear la necesidad de integrar de manera armoniosa todos los instrumentos del mix de comunicación en un conjunto unificado de manera que con independencia de las herramientas o medios a utilizar la marca "hable" una sola voz. Entre los factores que contribuyeron a que surgiera y se desarrollara la CIM cabe destacar, en primer lugar, el hecho de que la competencia en los mercados se hace cada vez más feroz y la diferenciación de los productos desde un punto de vista funcional es cada vez más escasa y duradera, requiriéndose cada vez más una diferenciación no funcional en términos de imagen y reputación de la marca. En segundo lugar, la creciente adopción de orientaciones estratégicas centradas en el mercado y el establecimiento de relaciones a largo plazo, lleva a que las empresas necesiten mantener una comunicación más fluida y frecuente con sus targets y a tener que integrar sus esfuerzos a fin de alcanzar sinergias comunicativas. Tercero, la progresiva fragmentación de las audiencias lleva a repartir las inversiones en comunicación en un número mayor de medios y soportes que obligan a intensificar los esfuerzos de coordinación. Finalmente, y en cuarto lugar el rápido desarrollo de las tecnologías asociadas a las bases de datos permite a las empresas crear, integrar y gestionar bases de clientes con las que dirigir mensajes directos e interactivos.

Con todo esto la CIM va ganando protagonismo entre las empresas y dentro del mundo académico. Así, en un estudio realizado por la Promotion Marketing Association of America en 1993 entre los 100 principales ejecutivos de marketing americanos se concluyó que la CIM sería el factor más determinante en la implementación de la estrategia de marketing en el futuro. Por otro lado, autores como Schultz et al. (1994) llegaron a afirmar que la CIM sería la única ventaja competitiva sostenible de marketing en las organizaciones a lo largo de la década de los 90 y dentro del siglo XXI. En esta misma línea, diversas investigaciones empíricas han corroborado un uso cada vez más extendido en la práctica de la CIM (Caywood, Schultz y Wang, 1991; Phelps, Harris y Johnson, 1996).

Independientemente de su tasa de implantación actual en la empresa, es una realidad que la CIM está siendo cada vez más aceptada y tenida en cuenta y que hoy en día existe ya una cierta consciencia sobre la "marca CIM" (Kitchen, Brignell, Li y Spickett-Jones, 2004; Kim, Han y Schultz, 2004).

Sin embargo, una importante barrera para su desarrollo definitivo lo constituye la ausencia de una definición universalmente aceptada sobre lo que es y representa la CIM en la realidad empresarial actual. Su debate conceptual está aún presente en la literatura académica como lo muestra el hecho de que buena parte de los trabajos publicados hasta la fecha se hayan centrado en su delimitación 
conceptual. Está aún por aclararse si la CIM se debe centrar únicamente en la integración de las distintas herramientas de comunicación al objeto de conseguir alcanzar sinergias de comunicación (perspectiva reducida), o debe incluir además la integración de todas las formas de comunicación inter e intra-organizacional enviadas tanto a audiencias internas como externas (perspectiva ampliada). Este debate acerca de su definición no sólo está presente a nivel académico sino que también es una realidad a nivel profesional (Swain, 2004; Gould, 2004).

A pesar del amplio cuerpo teórico que existe acerca del significado de la CIM y su papel en la gestión corporativa y de marketing apenas si se han desarrollado intentos para medirla y así comprobar el verdadero impacto que produce en el mercado. Así pues, el objetivo de este trabajo es examinar los principales intentos que se han producido hasta la fecha para medir el grado de integración de la comunicación alcanzado por una organización y proponer una escala de medida que recoja las diferentes dimensiones que componen la CIM de acuerdo al debate conceptual existente.

\section{Intentos de medida de la CIM}

A lo largo de la última década algunos autores han desarrollado varias herramientas para medir el grado de implantación de la CIM en las organizaciones. A continuación, se hace un repaso de las más relevantes.

\subsection{La aproximación de Phelps y Johnson}

Phelps y Johnson (1996) realizaron el primer intento serio para medir la CIM como concepto multidimensional. Para desarrollar su escala de medida estos autores partieron de las tres dimensiones propuestas por Nowak y Phelps (1994) en un estudio previo: one voice (una única voz), comunicaciones integradas, y campañas de comunicación coordinadas; al considerar que estas tres dimensiones reflejan el sentir de la profesión y forman parte del significado subyacente de la CIM. En la elaboración de su herramienta de medida de la CIM dedicaron los 4 primeros ítems a la medición de las dimensiones one voice y comunicaciones integradas. Dichos ítems estaban relacionados con la valoración de la coherencia y claridad de los mensajes (ítem 1: 'un único mensaje'), de la existencia de una estrategia común que unifique la actividad de todas las herramientas de marketing (ítem 2: 'estrategia común'), y del mantenimiento de una imagen coherente a través de las relaciones públicas (ítem 3: 'comportamiento e imagen de RRPP') y de la publicidad (ítem 4: 'comportamiento e imagen de publicidad'). La tercera dimensión, campañas de comunicación coordinadas, asocia la integración con la coordinación y propusieron 2 ítems para su medida (ítem 5: objetivos comunes, ítem 9: públicos-objetivos). Desde esta perspectiva, los autores proponen que la CIM significa "coordinar las disciplinas de comunicación de marketing o instituir los pasos que dan como resultado una mejor coordinación entre funciones y/o agencias".

Además de los 7 ítems anteriores, Phelps y Johnson (1996) incluyeron otros 14 ítems susceptibles de medir y despejar nuevas dimensiones de la CIM. 
Desarrollada la escala, analizaron sus propiedades psicométricas utilizando para ello una muestra de profesionales de marketing y la comunicación. Los resultados del análisis factorial llevado a cabo les permitió extraer finalmente cinco dimensiones subyacentes a la CIM, en orden de representatividad:

1) marketing directo,

2) one voice (una única voz),

3) coordinación de las campañas de comunicación,

4) responsabilidad incrementada,

5) objetivo respuesta.

La dimensión "marketing directo" destacaba como la más relevante en la medida de la CIM. Sin lugar a dudas, la implementación de un programa de CIM está fuertemente relacionada con el desarrollo de las bases de datos. En este sentido, Duncan (2005) afirma que las bases de datos son esenciales para las empresas de cara a personalizar los mensajes y empezar a moverse de forma intencional hacia una comunicación realmente bidireccional. Sin las bases de datos la interactividad, elemento crucial de la CIM se perdería. Los resultados del estudio confirmaron las dimensiones "one voice” y "coordinación de las campañas de comunicación" como básicas dentro de la estructura subyacente al significado de CIM. Por su parte, la dimensión "responsabilidad incrementada" emerge como cuarto factor. El aumento de responsabilidad en una determinada área de comunicación no implica una disminución de responsabilidad en otra, sino coincide con un aumento de responsabilidad similar. El último factor identificado es el "objetivo respuesta", los objetivos de la CIM son respuestas tangibles de su implementación, es decir aumento de las ventas o de consciencia de marca. Este factor no aporta novedades con respecto a los objetivos del marketing, sin embargo, representa una novedad en cuanto a los medios a través de los cuales alcanzarlos.

\subsection{El intento de medida de Low}

Low (2000) realizó un estudio basado en la definición de Duncan y Everett (1993), según la cual la CIM es "la coordinación estratégica de todos los mensajes y medios utilizados por una organización para influir en su valor de marca percibido". Este autor basó su estudio en una entrevista a 15 gestores de marketing expertos (senior marketing managers) a quienes les solicitaba que definieran los que entendían por CIM. Observó que el elemento más presente en las definiciones de los profesionales resultó ser la coordinación de las herramientas de comunicación de marketing, lo que proporcionaba un apoyo adicional para la elección de la definición de Duncan y Everett (1993).Los entrevistados sugirieron también cuatro componentes nuevas que contribuían a la coordinación de las actividades de comunicación de marketing:

1) Planificar y ejecutar diferentes herramientas de comunicación (publicidad, promoción de ventas, relaciones públicas y mail directo) como un único proyecto integrado.

2) Asignar la responsabilidad de todos los esfuerzos de comunicación a un único director.

3) Asegurar que los distintos elementos del programa de comunicación tienen un objetivo común estratégico (por ejemplo, reforzar una particular estrategia de posicionamiento). 
4) Centrarse en un mensaje de comunicación común.

Finalmente, con toda esta información Low (2000) desarrolló una medida de la CIM compuesta por 3 ítems medidos en una escala tipo Likert que hacen referencia a la centralización de la planificación y dirección de las herramientas de comunicación de marketing, a la coherencia estratégica de los elementos del programa de comunicación y finalmente, y al enfoque de las herramientas de comunicación en un mensaje común.

\subsection{La propuesta de Reid basada en el mini-audit de Duncan y Moriarty}

Duncan y Moriarty $(1997,1998)$ elaboraron uno de los intentos más completos hasta la fecha para medir la CIM, al que denominaron mini-audit. Se trata de una herramienta diagnóstica dirigida a ayudar a los directivos en la gestión de las áreas fuertes y débiles de la integración. El objetivo del mini-audit era la evaluación de las prácticas de CIM en la construcción de las relaciones con los clientes. Gracias al análisis de la estructura organizativa y del grado de comprensión de los objetivos y estrategias de comunicación de marketing, esta herramienta mide el grado en que los mensajes de marca creados por la empresa son estratégicamente consistentes.

Esta herramienta de medida fue desarrollada siguiendo la teoría según la cual existen 10 factores que contribuyen a la formación de relaciones de marca que pueden agruparse en tres categorías:

1) Factores relacionados con la creación y el fomento de las relaciones con todos los grupos de interés, stakeholders, y no sólo con los clientes;

2) Factores relacionados con el proceso de integración: la coherencia estratégica, la interactividad intencional, la misión de marketing y la planificación llamada 'zero-based planning';

3) Factores relacionados con la organización: la gestión interfuncional, las competencias centrales, el marketing de bases de datos y la colaboración con una agencia integrada.

Unos años más tarde, Reid (2005) adaptó el mini-audit de Duncan y Moriarty $(1997,1998)$ para construir una herramienta modificada que no sólo sirviera como ‘diagnóstico' del nivel de integración de una empresa, sino que también permitiera medir los efectos del proceso de CIM en los resultados de la marca. El objetivo de este estudio era proveer un análisis exploratorio de la relación existente entre el grado de CIM alcanzado en la organización y los resultados alcanzados. Reid (2005) concluyó que los constructos normativos de la CIM teorizados por Duncan y Moriarty (1997) podrían resumirse en los tres siguientes: interactividad, misión de marketing y planificación estratégica interfuncional (que engloba infraestructura organizativa, consistencia estratégica, planificación y evaluación). La escala final propuesta por Reid (2005) incluía un total de 20 ítems medidos a través de una escala tipo Likert, donde los dos primeros tratan de medir el grado de interactividad alcanzado en la empresa a través de la medición de la capacidad de la empresa de elaborar programas especial para atender las consultas y quejas de los clientes y almacenarlas en bases de datos. La integración de la misión de marketing en la comunicación de la organización, otro elemento clave de la CIM, se valoraba a través 
de otros dos ítems que pretendían medir el nivel de importancia del eslogan de la misión en la planificación de la comunicación y su grado de promoción entre clientes y otros grupos de interés. Los restantes ítems trataban de medir las tres dimensiones de la planificación estratégica interfuncional. En concreto, tres ítems medían distintos aspectos de la infraestructura organizativa: el grado en el que el personal que gestiona el programa de comunicación conoce las debilidades y fuerzas de todas las herramientas de comunicación de marketing, el nivel de calidad de la comunicación interna y de las relaciones recíprocas entre las agencias externas de comunicación. La coherencia estratégica se evaluaba por medio de tres ítems más: grado de actualización del plan de marketing, nivel de flexibilidad promocional, y coordinación de los mensajes enviados a través de todas las operaciones (por ejemplo, los precios, la distribución etc.).

Finalmente, emplea el mayor número de ítems (5) para la valoración de los aspectos relativos a la planificación y evaluación de la comunicación. En detalle, estos ítems consideran la realización de un análisis DAFO y la prioridad de la gestión de los contactos de los clientes con el firme objetivo de crear y mantener relaciones rentables con clientes y otros stakeholders (dentro de la planificación de la comunicación) como determinantes para la obtención de un alto nivel de integración. Además, el uso de un estudio sistemático para estimar la fuerza de las relaciones con los grupos de interés, la presencia de estrategias que maximicen las fuerzas distintivas de las distintas herramientas de comunicación de marketing representan importantes elementos-clave de la CIM, según este autor.

En su investigación empírica Reid (2005) validó, utilizando una muestra importante de empresas y diferenciada por sectores de actividad, la escala propuesta y obtuvo que la principal dimensión que contribuye al grado de integración de la comunicación fue la gestión interfuncional, seguida de la interactividad y en último lugar de la misión de marketing.

\subsection{La herramienta de medición de Lee y Park}

La propuesta más reciente hasta la fecha de medición de la CIM es la que llevan a cabo Lee y Park (2007). Estos autores plantean una conceptualización teórica del constructo basada en cuatro dimensiones:

1) Comunicaciones unificadas para un mensaje y una imagen coherentes.

2) Comunicaciones diferenciadas para los diversos grupos de clientes.

3) Comunicaciones dirigidas por bases de datos para resultados tangibles.

4) Comunicaciones que fomenten las relaciones con los clientes existentes.

Estos autores elaboraron una escala exhaustiva y a la vez parsimoniosa de 18 ítems al objeto de medir las actividades más relevantes dentro de cada dimensión, siguiendo una metodología rigurosa, y mostrando una fiabilidad estable y una validez robusta. Esta herramienta no mide sólo las cuatro dimensiones centrales de la CIM, sino que capta además aspectos clave de cada dimensión con múltiples ítems.

La dimensión comunicaciones unificadas para un mensaje y una imagen coherentes medía si el mensaje deseado se comunica de forma coherente con todas las herramientas y todos los canales de 
comunicación. Dentro de esta dimensión la escala pretende estimar si la coherencia visual y lingüística se mantiene en la comunicación de los mensajes y si las actividades de comunicación de la empresa son llevadas para crear y mantener una imagen de marca coherente.

La segunda dimensión, comunicaciones diferenciadas para grupos de múltiples clientes, además de detectar el grado en que la empresa decide si es necesario crear múltiples imágenes de marca para distintos públicos en el mercado-objetivo, también se propone estimar de qué manera la empresa utiliza la información del consumidor para desarrollar sus programas de comunicación para múltiples públicos.

A través de la tercera dimensión, comunicaciones dirigidas por bases de datos para resultados tangibles, la escala tiene como objetivo medir cómo las actividades de comunicación de marketing de la empresa generan respuestas de comportamiento en el cliente. Además, esta dimensión detecta si la empresa recoge e integra la información del cliente en una base de datos.

Finalmente, esta escala no sólo abarca importantes actividades de la CIM identificadas en estudios anteriores, sino que incluye una dimensión nueva: las comunicaciones que fomentan la relación con los clientes existentes. Su objetivo es medir el grado en que las actividades de comunicación de la empresa son programadas para reforzar las relaciones con los clientes existentes. Además, a través de esta dimensión, la escala es susceptible de estimar si las actividades de comunicación centradas en la relación son diseñadas para producir rentabilidad a largo plazo.

Tras su investigación empírica estos autores obtienen que esta escala posee buenas propiedades psicométricas para medir la CIM.

\section{Propuesta de medida de la CIM}

La falta de una definición universalmente aceptada representa la barrera más fuerte para el desarrollo de herramientas de medida de la CIM. No se puede medir la CIM sin antes especificar qué es y cuáles son sus principales dimensiones. En este sentido, se propone la siguiente definición:

"Proceso interactivo y sistémico de planificación interfuncional y optimización de los mensajes enviados a todos los grupos de interés de cara a comunicar con coherencia y transparencia al objeto de conseguir sinergias y fomentar relaciones rentables a largo plazo".

Esta definición recoge el carácter multidimensional del concepto, distinguiéndose cuatro dimensiones principales:

1) One voice, una única voz.

2) Interactividad.

3) Planificación interfuncional.

4) Relaciones rentables a largo plazo. 
Se ha desarrollado una herramienta, compuesta por 23 ítems, (ver Tabla 1) de cara a representar las distintas facetas de las cuatro dimensiones de la CIM, partiendo de la dimensión más básica (one voice) hasta llegar al aspecto más complejo que representa el objetivo final del paradigma de la CIM: relaciones rentables a largo plazo.

Los primeros 5 ítems de la escala pretenden medir el nivel de coherencia de los mensajes y de la imagen y el grado de coordinación de las herramientas de comunicación en el marco de una estrategia común. Todos estos aspectos forman parte de la dimensión que Nowak y Phelps (1994) definieron como 'one voice' y que según ellos representa la esencia de la integración.

Los 6 ítems siguientes tratan de medir la interactividad. La CIM implica una comunicación en una doble vía, puesto que a través de este nuevo paradigma se busca mantener un diálogo continuo entre la empresa y sus stakeholders. En este sentido, la innovación tecnológica desempeña un papel muy relevante. Diversos autores (Schultz y Schultz, 1998; Duncan y Moriarty, 1997) consideran que las bases de datos e Internet resultan ser especialmente relevantes para la implementación de la CIM, puesto que refuerzan la validez del enfoque, la rigurosidad del método y la eficacia de su uso. Además, las herramientas de marketing directo desempeñan un papel destacado para la puesta en marcha de una comunicación bidireccional entre la empresa y sus grupos de interés.

En la comunicación de marketing tradicional el punto de referencia es el mix de comunicación, en cambio el paradigma de la CIM se centra en la empresa en su totalidad. No sólo el departamento de marketing envía mensajes, toda la empresa en su conjunto es una fuente constante de emisión de mensajes que es preciso integrar para conseguir "hablar una única voz". Aunque no se quiera, no podemos dejar de comunicar (you cannot not communicate) de ahí que la CIM implique un importante esfuerzo en planificación y gestión interfuncional. Esta es precisamente la tercera dimensión del concepto, que la escala propuesta pretende medir a través de siete ítems (12 al 18). La comunicación interna, especialmente la comunicación horizontal, la coherencia estratégica y la planificación basada en objetivos representan las facetas más destacadas de esta dimensión.

Puesto que la CIM debe representar una herramienta fundamentalmente estratégica, ésta debe proponer la consecución de relaciones rentables a largo plazo. Los últimos 5 ítems (19 al 23) pretenden medir este aspecto que constituye la cuarta y última dimensión propuesta y hacen referencia a aspectos relacionados con la gestión de los contactos, el fomento de la satisfacción y la lealtad de los clientes, la planificación y la evaluación continua de la calidad y la rentabilidad de las relaciones de las empresas con sus stakeholders.

\section{Conclusiones}

Después de más de una década de intensa investigación académica y debates, hoy en día la CIM representa uno de los conceptos más utilizados en el ámbito de la comunicación de marketing. A pesar de su creciente popularidad, sigue habiendo ciertas deficiencias en las bases teóricas del paradigma de la CIM. Además, su impacto en la performance de marketing no ha sido contrastado adecuadamente a nivel empírico, debido a la ausencia de herramientas de medida válidas del constructo. 
En este trabajo, tras una revisión exhaustiva de la literatura se propone una definición de la CIM y se identifican cuatro dimensiones que subyacen al concepto. Finalmente, se diseña una herramienta para medir el grado de integración que presenta una empresa. En definitiva, esta investigación representa un primer paso importante para hacer frente al problema de la medición de la CIM, que actualmente constituye el reto más ambicioso de la investigación acerca del paradigma de la CIM.

Alentamos a la investigación académica a proponer alternativas a esta herramienta de medida así como a tratar de validarla empíricamente recurriendo para ello a muestras representativas de directores de marketing y comunicación por sectores de actividad.

\section{Tabla 1. Herramienta de medida de la CIM}

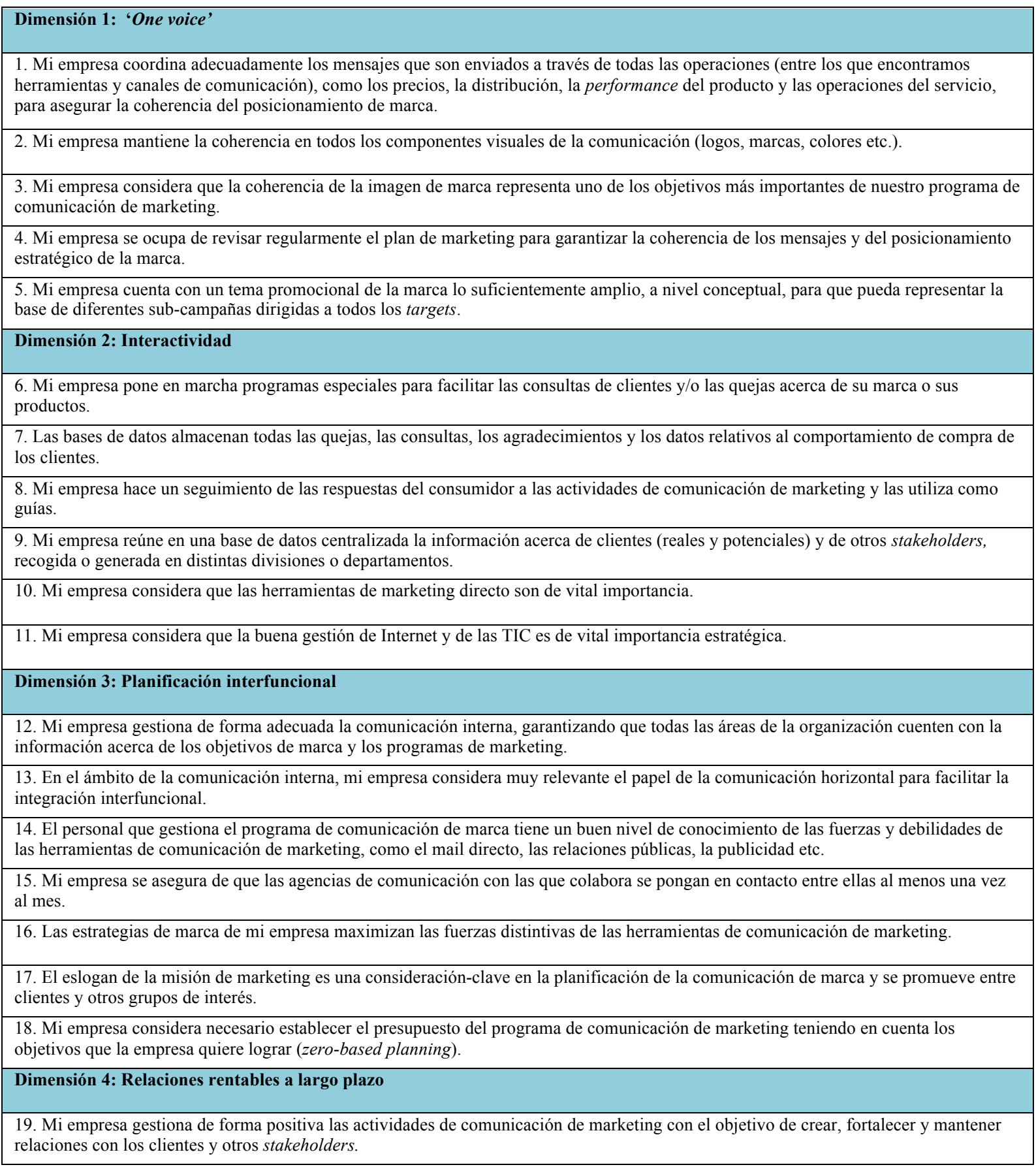




\begin{tabular}{|l|}
\hline $\begin{array}{l}\text { 20. Mi empresa pone en marcha actividades dirigidas a generar un flujo continuo de beneficios de clientes individuales para fomentar } \\
\text { relaciones a largo plazo con los mismos. }\end{array}$ \\
\hline 21. Mi empresa tiene como objetivo prioritario el aumento del nivel de satisfacción de los clientes de cara a conseguir su fidelización. \\
\hline $\begin{array}{l}\text { 22. Mi empresa elabora y pone en marcha algún tipo de estudio sistemático para evaluar la calidad de sus relaciones con todos los } \\
\text { stakeholders. }\end{array}$ \\
\hline 23. En el plan anual de comunicación de marketing, la prioridad es la gestión de los contactos con los stakeholders.
\end{tabular}

Fuente: Elaboración propia a partir de Phelps y Johnson (1996), Duncan y Moriarty (1997), Low (2000), Reid (2005), Lee y Park (2007). Escala Tipo Likert de 7 puntos (1: totalmente en desacuerdo, 7: totalmente de acuerdo)

\section{Referencias bibliográficas}

Caywood, C.; Schultz, D. E.; Wang, P. (1991), Integrated Marketing Communications: a survey of National Goods Advertisers, informe no publicado, Bloomington, IN: Medill School of Journalism, Northwestern University, June.

Duncan, T. y Everett, S. E. (1993), "Client Perceptions of Integrated Communications", Journal of Advertising Research, Vol.32, N.3, pp. 30-39.

Duncan, T. y Moriarty, S. E. (1997), Driving Brand Value. New York: McGraw Hill.

Duncan, T. y Moriarty, S. E. (1998), “A Communication-Based Marketing Model for Managing Relationships", Journal of Marketing, Vol.62, pp.1-13.

Gould, S. J. (2004), "IMC as Theory and as Poststructural Set of Practices and Discourses: A Continuously Evolving Paradigm Shift”, Journal of Advertising Research, Vol.44, March, pp.66-70.

Lee, D. H. y Park, C. W. (2007), "Conceptualization and Measurement of Multidimensionality of Integrated Marketing Communications”, Journal of Advertising Research, Vol. 47, pp. 222-236.

Low, G. L. (2000), “Correlates of Integrated Marketing Communications”, Journal of Advertising Research, Vol.40, N.3, pp. 27-39.

Kim, I.; Han, D.; Schultz, D. E. (2004), “Understanding the Diffusion of Integrated Marketing Communication", Journal of Advertising Research, Vol.44, March, pp.31-45.

Kitchen, P. J.; Brignell, J.; Li, T.; Spickett-Jones, G. (2004), "The Emergence of IMC: A Theoretical Perspective", Journal of Advertising Research, Vol.44, March, pp.19-30.

Nowak, G.; Phelps, J. (1994), “The integrated Marketing Communications Phenomenon: An examination of its impact on Advertising practices and its Implications for Advertising Research", Journal of Current Issues and Research in Advertising, Vol. 16, N.1, pp.49-66.

Phelps, J. E. y Johnson, E. (1996), “Entering the quagmire: examining the 'meaning' of integrated marketing communications", Journal of Marketing Communications, Vol. 2, N. 3, pp.159-172. 
Phelps, J.; Harris, T.E.; Johnson, E. (1996), "Exploring decision-making approaches and responsibility for developing marketing communications strategy", Journal of Business research, Vol. 37, N. 3, pp. 217-223.

Reid, M. (2005), "Performance Auditing of Integrated Communication (IMC) actions and outcomes", Journal of Advertising, Vol. 34, N.4, pp.41-54.

Schultz, Tannenbaumm y Lauterborn (1994), The new Marketing Paradigm. Integrated Marketing Communication, Chicago, IL: NTC Business Books.

Swain, W. N. (2004), "Perceptions of IMC after a Decade of Development: Who's at the Wheel, and How Can We Measure Success?", Journal of Advertising Research, Vol. 44, N. 1, pp.46-65. 